\title{
Tumor-associated macrophages, dendritic cells, and neutrophils: biological roles, crosstalk, and therapeutic relevance
}

https://doi.org/10.1515/mr-2021-0014

Received May 16, 2021; accepted August 3, 2021;

published online February 14, 2022

\begin{abstract}
Tumor-associated myeloid cells constitute a series of plastic and heterogeneous cell populations within the tumor microenvironment (TME), and exhibit different phenotypes and functions in response to various microenvironmental signals. In light of promising preclinical data indicating that myeloid-based therapy can effectively suppress tumor growth, a series of novel immune-based therapies and approaches are currently undergoing clinical evaluation. A better understanding of the diversity and functional roles of different myeloid cell subtypes and of how they are associated with TME remodeling may help to improve cancer therapy. Herein, we focus on myeloid cells and discuss how tumor cells can simultaneously reprogram these cells through tumor-derived factors and metabolites. In addition, we discuss the interactions between myeloid cells and other cells in the TME that have the potential to directly or indirectly regulate tumor initiation, invasion, or angiogenesis. We further discuss the current and future potential applications of myeloid cells in the development of focused therapeutic strategies in cancer treatment.
\end{abstract}

Keywords: cell-interaction; dendritic cells; macrophages; neutrophils; therapy; tumor microenvironment.

\footnotetext{
*Corresponding authors: Yanhua Du and Youqiong Ye, Department of Immunology and Microbiology, Shanghai Institute of Immunology, Shanghai Jiao Tong University School of Medicine, Shanghai 200025, China, E-mail: yhdu@shsmu.edu.cn (Y. Du), youqiong.ye@shsmu.edu.cn (Y. Ye). https://orcid.org/0000-00018332-4710 (Y. Ye)

Mingyi Shen, Department of Immunology and Microbiology, Shanghai Institute of Immunology, Shanghai Jiao Tong University School of Medicine, Shanghai, China
}

\section{Introduction}

Efforts to uncover the link between tumor cell genetic mutations and tumor progression have been ongoing for several decades. Recently, researchers have discovered that in addition to gene mutations, tumor progression is also regulated by tumor-associated immune and nonimmune cells [1]. The tumor immune microenvironment (TIME) includes immunosuppressive cells, such as tumorassociated macrophages (TAMs), myeloid-derived suppressor cells (MDSCs), and regulatory T (Treg) cells, as well as anti-tumor effector cells, such as cytotoxic $\mathrm{CD} 8^{+} \mathrm{T}$ cells, $\mathrm{CD}^{+}{ }^{\mathrm{T}}$ helper (Th), and natural killer (NK) cell. The tumor microenvironment (TME) also contains a variety of nonimmune cell populations such as endothelial cells and fibroblasts [2], with all cells in this environment exhibiting substantial diversity and plasticity.

The discovery of the cell-cell interaction in the TME has led to a revolution in cancer treatment, from the development of chemotherapy and radiation strategies that target tumor cells to the design of antibody-based immunotherapies that modulate immune responses [3]. As central mediators of adaptive immunity, $\mathrm{T}$ cells represent effective targets for immunotherapy. Immune checkpoint blockade (ICB) therapies targeting programmed death 1 (PD-1) and cytotoxic T-lymphocyte antigen 4 (CTLA-4) on the surface of $\mathrm{T}$ cells have greatly prolonged cancer patient survival [4]. However, the number of patients who respond to these types of therapies is still limited, leading to efforts to develop new types of inhibitors. Several subtypes of myeloid cells, including macrophages, dendritic cells (DCs), and MDSCs can promote tumor development by producing tumorpromoting factors and molecules that inhibit the cytotoxic activity of $\mathrm{T}$ cells. Specific myeloid cell subsets also express PD-1, and some myeloid-specific immune checkpoint molecules have additionally been discovered [5]. Therein, the checkpoints on macrophage, for example, signal regulatory protein alpha (SIRP $\alpha$ ) and colony stimulating factor 1 receptor (CSF1R), have undergone decades of research. The results show the checkpoints contribute 
to the immunosuppressive function of TAMs and their therapeutic antibodies are currently being tested in clinical trials [6]. Moreover, based on the DCs antigen presentation function, DCs activating and motivating factors can be administered, leading to anti-tumor function [7]. These suggest that myeloid cells may represent potential therapeutic targets. In additional to the most studied macrophages and DCs, the importance of neutrophils in cancer has been increasing apparent recently and some neutrophils-specific therapeutic methods occur. Thus, we mainly focus on tumorassociated macrophages, DCs, and neutrophils in this review. All three cell types undergo the development from haematopoietic stem cells to tumor-infiltrating myeloid cells, which is regulated by tumors [1]. They exhibit a diverse array of phenotypes that can promote or suppress tumor growth and function, and they play important regulatory roles through their crosstalk with tumor cells, T cells, and other cell populations. A comprehensive understanding of the relationships among tumor-infiltrating myeloid cells and other populations in the TME is critical in order to provide important insights into the mechanisms underlying immune surveillance and tumor immunotherapy.

In this review, we focus on the roles of macrophages, DCs, and neutrophils, and we discuss the mechanisms whereby tumor cells regulate myeloid cells and promote their metabolic reprogramming. We then discuss the interactions between myeloid cells and other cells in the TME to highlight their crucial roles in tumor progression. Finally, we explore current therapeutic strategies targeting myeloid cells that may be beneficial in overcoming an immunosuppressive TME to promote $\mathrm{T}$ cell-mediated tumor clearance.

\section{Tumor-associated macrophages}

Macrophages represent a versatile subset of myeloid immune cells that execute a broad array of functions, including the regulation of tissue homeostasis, defenses against pathogens, and the promotion of wound healing [8]. Macrophages that infiltrate tumors or populate the TME of solid tumors are defined as TAMs, and can affect tumor growth, tumor angiogenesis, immune regulation, metastasis, and chemoresistance.

\section{Phenotypic properties and functional roles of TAMs}

Circulating monocytes derived from bone marrow hematopoietic stem cells (HSCs) are generally believed to be the primary source of macrophages. However, recent evidence suggests that macrophages are maintained in most healthy tissues by embryonic precursors independently of monocytes $[9,10]$. The tissue-resident macrophages (TRMs) of embryonic origin and circulating monocytes are two main sources of TAMs during tumor progression. In pancreatic ductal adenocarcinoma mouse model, TAMs of different origins show various impacts on fibrosis, herein, embryonically derived TAMs have a fibrotic phenotype with higher expression of molecules involved in extracellular matrix deposition and remodeling [11]. Although the origins of macrophages have been mapped in multiple animal models, our ability to interrogate these populations in human tissues remains limited. As these technologies continue to develop, RNA velocity analyses embedded on a diffusion map can be used to infer the future fate of human cell populations. For example, in colorectal cancer (CRC) patients, a strong directional flow from $\mathrm{CD} 14^{+}$monocytes towards macrophage populations has been reported based on RNA velocity analysis. Monocytes give rise to TAMs through different tissue-resident macrophages [12]. RNA velocity analyses can be expanded to other cancers, and have the potential to permit future analyses of macrophage origins [13].

Activated macrophages are often classified into the M1 (classically activated) and M2 (alternatively activated) subtypes [14]. In general, M1 macrophages promote inflammatory responses against invading pathogens and tumor cells, whereas M2 macrophages tend to exhibit an immunosuppressive phenotype, favoring tissue repair and tumor progression. The conversion between M1 and M2 macrophage subtypes is a dynamic process known as macrophage polarization that occurs in response to microenvironmental signals. Based on their functions within the tumor microenvironment, TAMs are most often characterized as M2-like macrophages, given that they express higher levels of anti-inflammatory cytokines, scavenging receptors, angiogenic factors, and proteases than do M1-type macrophages [15]. However, it is worth noting that M1 and M2 types are just relative terms and macrophages actually consist of a continuum of phenotypes. For example, although TAMs are conventionally acknowledged as M2-like macrophages, to be exact, they exhibit phenotypes anywhere in between M1 and M2.

In a recent systemic analysis of myeloid cells across 15 human cancer types, macrophages subsets in different tumor types showed heterogeneous transcriptomic patterns [16]. TAM subsets were identified with various marker genes, including $S P P 1, C 1 Q C$, ISG15, and $F N 1$, and different TAM subsets presented different functional phenotypes. For example, $S P P 1^{+}$TAMs in CRC patients had highly correlation with angiogenesis, leading to poor prognosis and resistance 
to CSF1R treatment [12]. With more and more TAMs subsets being discovered, some of them dash the stereotype as playing pro-tumor roles. The complement activation and antigen processing and presentation pathways significantly increase in $\mathrm{ClOC}^{+}$TAMs [12]. Meanwhile, $\mathrm{CxCl}^{+}$TAMs in CT26 colorectal carcinoma facilitate the recruitment of protective $\mathrm{CxCr}^{+} \mathrm{T}$ cells and predict the response to anti-PD-L1 treatment [17]. Since the functions of these subgroups are mainly based on bioinformatics analysis and the limitations of experimental support, we still focus on the function of TAMs to stimulate tumor progression in this review.

\section{Tumor cell-mediated regulation of TAMs polarization and function}

\section{The regulatory roles of tumor-derived factors}

Several factors produced by tumor cells can influence macrophage polarization (Figure 1A and Table 1). Tumorderived colony stimulating factor 1 (CSF1), a primary chemoattractant and functional regulator for macrophages, functions in synergy with interleukin (IL)-4 to drive the polarization of M2 macrophages [18]. Microparticles are specialized subcellular vesicles from 100 to $1,000 \mathrm{~nm}$ in diameter, and macrophage uptake of tumor-derived microparticles can drive their M2 polarization and the apoptotic death of M1 macrophages, promoting tumor growth and metastasis [19]. Furthermore, hypoxia shapes and induces specific macrophage phenotypes that support tumor malignancy, given that hypoxia promotes immune evasion, angiogenesis, tumor cell survival, and metastatic dissemination. In the hypoxic TME, the cytosolic accumulation of hypoxiainducible factors $1 \alpha$ (HIF-1 $\alpha$ ) induces secretion of high mobility group box-1 (HMGB1) by melanoma tumor cells and promotes M2-like macrophage accumulation [20]. Hypoxia-primed cancer cells can also attract and polarize macrophages towards a pro-angiogenic M2-polarized subtype via the release of eotaxin and oncostatin M (OSM) [21]. Hepatocellular carcinoma (HCC) progression can be accelerated via the upregulation of HIF-1 $\alpha$-mediated IL-10, promoting M2 macrophage polarization [22]. Hypoxia can further induce the production of key monocyte recruitment factors including C-C motif chemokine ligand (CCL) 2, CCL5, CXC-chemokine ligand 12 (CXCL12), CSF1, and vascular endothelial growth factor (VEGF) by tumor cells and the stroma [23]. Once recruited into hypoxic regions, monocytes downregulate the receptors for several of these factors, effectively trapping differentiated TAMs within hypoxic microenvironments [24].

\section{Metabolic regulation}

One of the TCA cycle metabolites, $\alpha$-ketoglutarate ( $\alpha$-KG), induces macrophage polarization towards an $\mathrm{M} 2$ phenotype (Figure 1A). Inhibiting glutaminase, which provides glutamate as a source for $\alpha$-KG production, decreases M2 polarization while increasing M1 polarization. A recent study found that $\alpha-K G$ supports $\mathrm{M} 2$ activation by promoting the Jmjd3-dependent demethylation of histone 3 at lysine 27 (H3K27) on the promoter regions associated with M2-specific marker genes [25]. While $\alpha$-KG serves as a co-stimulator for Jmjd3, succinate serves as an inhibitor thereof such that the $\alpha-K G /$ succinate ratio determines macrophage polarization status. While an increased $\alpha-\mathrm{KG} /$ succinate ratio favors $\mathrm{M} 2$ polarization, a decreased $\alpha$-KG/succinate ratio induces cells to undergo differentiation towards the proinflammatory M1 macrophage phenotype [25].

Adenosine is a nucleoside that can be released from tumor cells in the TME. Adenosine regulates the phagocytic activity of mononuclear cells, including macrophages, via the adenosine receptors (ADORA)1, 2A, 2B, and 3, which are G-protein-coupled transmembrane receptors. The dominant ADORA inducing M2 macrophage polarization is ADORA 2A, and based on the recent results, ADORA 2B also show this function [26]. Deletion of the ADORA 2A on macrophages favors M1 polarization and substantially reduces antiinflammatory IL-10 production [27]. Thus, adenosine in the TME leads to immunosuppression.

The signaling functions and polarization of M2 macrophages can also be regulated by lactate in the TME. Lactate is an end-product of aerobic glycolysis, which is highly active in tumor cells. Studies demonstrate that tumor cellderived lactate induces the expression of VEGF and the M2 polarization of TAMs via HIF1 $\alpha$ [28]. Sensing of lactate by macrophages in the context of M2 polarization is mediated by G-protein-coupled receptor 132 (Gpr132), and a loss of Gpr132 in mice inhibits breast cancer metastasis [29]. Additionally, decreased Gpr132 expression in patients with breast cancer is correlated with improved metastasis-free survival.

\section{TAMs promote tumor progression}

As mentioned above, TAMs are influenced by tumor cells in many ways. In turn, TAMs influence a number of critical biological functions that are linked with tumor progression. Here, we primarily focus on the correlation between TAMs and tumor invasion/angiogenesis (Figure 1B). Other aspects have been reviewed in detail previously [30, 31]. 
A

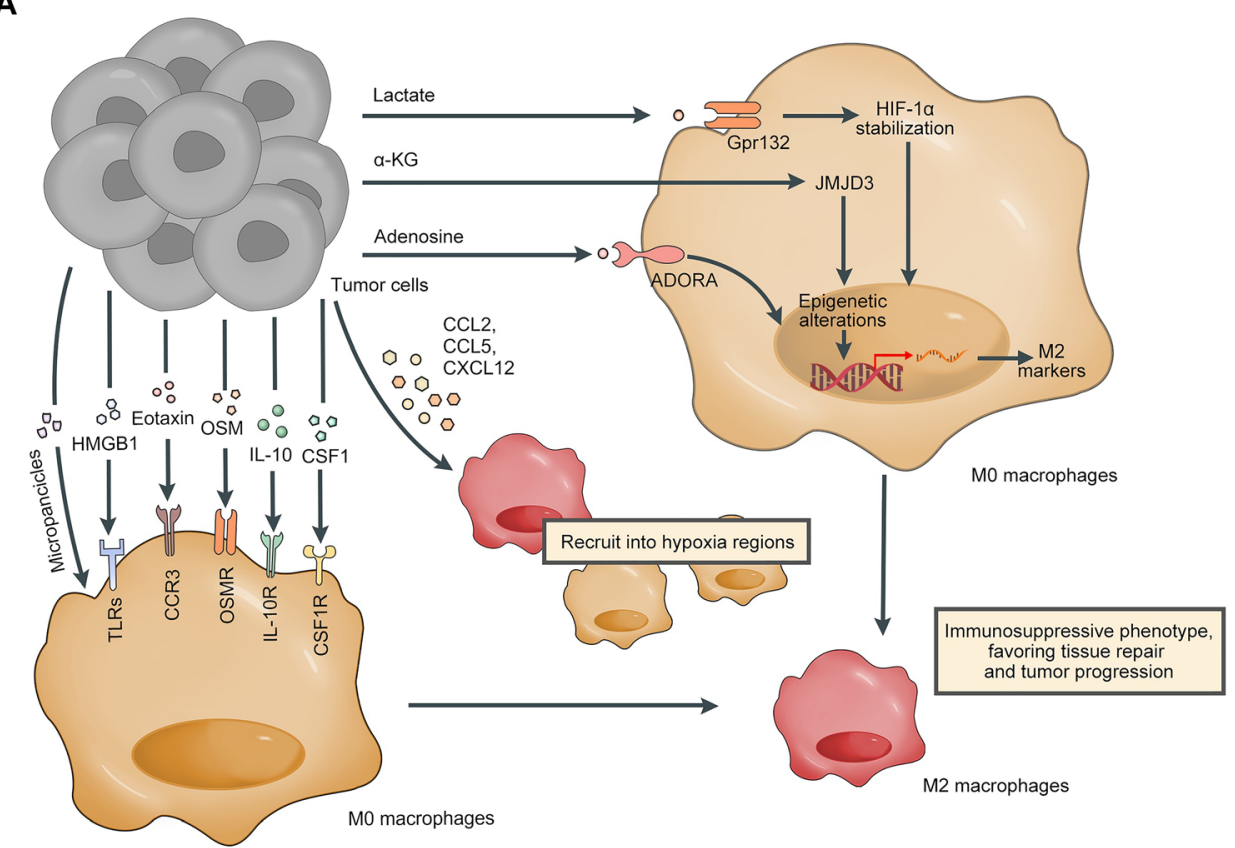

B

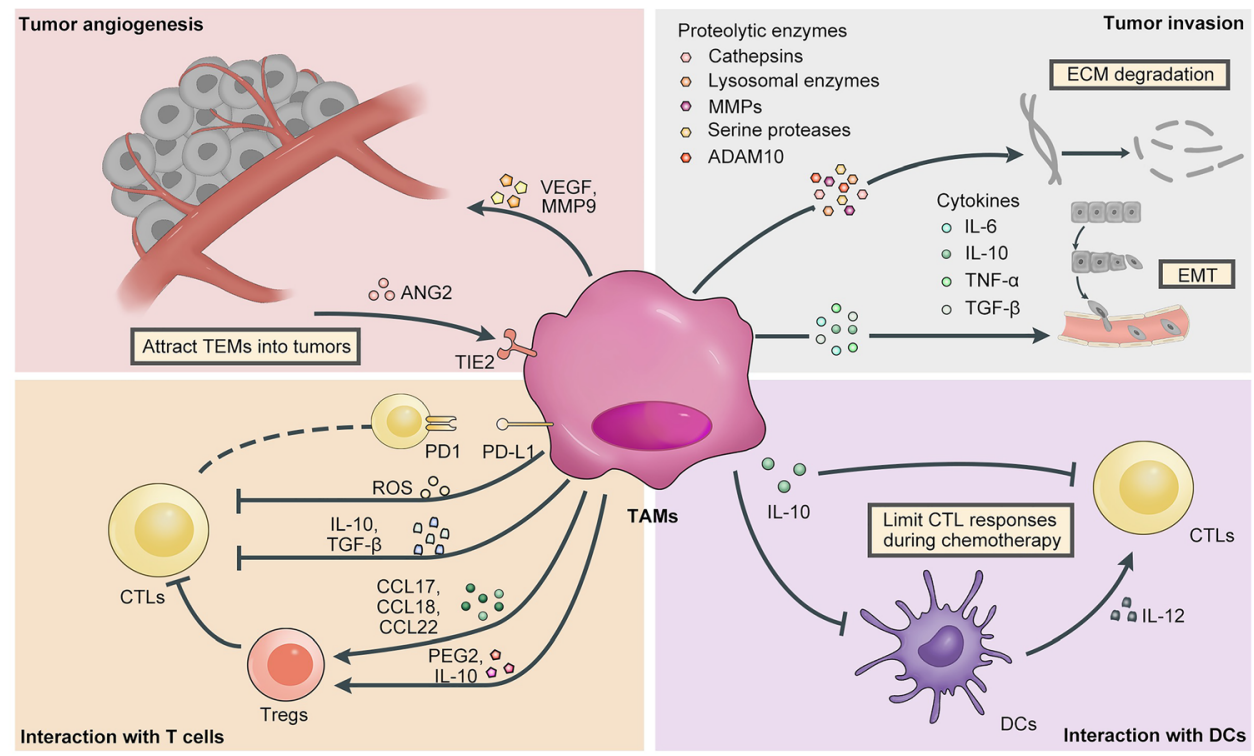

Figure 1: The roles of TAMs in tumor immunity.

(A) The polarization of M2 by tumor cells-derived factors and metabolites. To regulate TAM M2 polarization, tumor cells can secrete factors (CSF1, microparticles, HMGB1, Eotaxin, OSM and IL-10), interacting with corresponding receptors on macrophages. Tumor cells-derived chemokines (CCL2, CCL5, CXCL12) can recruit macrophages and trap them in hypoxic tumor region. Metabolites, including lactate, $\alpha-K G$ and adenosine, control polarization of M2 macrophages in different ways. (B) TAMs interact with other cells in TME. TAMs play particular functional roles in tumor progression, including tumor invasion (grey) and tumor angiogenesis (red). TAMs release inflammatory factors (IL-6, IL-10, TNF- $\alpha$ and TGF- $\beta$ ) to promote EMT and proteolytic enzymes (cathepsins, lysosomal enzymes, MMPs, serine proteases and ADAM10) to remodel ECM. For tumor angiogenesis, TAMs release a panel of pro-angiogenic factors (VEGF, MMP9, etc.) and endothelial cell-produced ANG2 can attract TEMs into tumors. TAMs also regulate T cells (orange) and DCs (purple) to induce immune suppression. CSF1, colony stimulating factor 1; HMGB1, high mobility group box-1; TLRs, toll-like receptors; OSM, oncostatin M; CCL, C-C chemokine ligand; CCR, C-C chemokine receptor; CXCL, CXC chemokine ligand; CXCR, CXC chemokine receptor; Gpr132, G-protein-coupled receptor 132; $\alpha$-KG, $\alpha$-ketoglutarate; ADORA, adenosine receptors; TNF- $\alpha$, tumor necrosis factor- $\alpha$; TGF- $\beta$, transforming growth factor- $\beta$; MMPs, matrix metalloproteinases; ADAM10, disintegrin and metalloproteinase domain-containing protein 10; ANG-2, angiopoietin-2; Tie2, tyrosine kinase receptor 2; VEGF, vascular endothelial growth factor; TEMs, Tie2-expressing monocytes; ROS, reactive oxygen species; PGE2, prostaglandin E2; PD-1, programmed cell death protein 1; PD-L1, programmed cell death 1 ligand 1. 
Table 1: Tumor-derived molecules related to polarization process of macrophages, DCs and neutrophils.

\begin{tabular}{|c|c|c|c|c|}
\hline Class & & Molecules & Description & Ref. \\
\hline \multirow[t]{9}{*}{ Macrophages } & Factors & CSF1 & M2 macrophage polarization can be induced in CSF1 and/or IL-4-dependent manner & [18] \\
\hline & & Microparticles & $\begin{array}{l}\text { Microparticles uptake leads to M2 polarization and the apoptotic death of M1 mac- } \\
\text { rophages, promoting tumor growth and metastasis }\end{array}$ & [19] \\
\hline & & HMGB1 & $\begin{array}{l}\text { Hypoxia induces the secretion of HMGB1 and promotes M2 macrophages } \\
\text { accumulation }\end{array}$ & {$[20]$} \\
\hline & & Eotaxin, OSM & Eotaxin and OSM are released by hyopxia cancer cells and induce M2 polarization & {$[21]$} \\
\hline & & IL-10 & HIF-1 $\alpha$-mediated IL-10 can promote M2 polarization & {$[22]$} \\
\hline & & $\begin{array}{l}\text { CCL2, CCL5, } \\
\text { CXCL12 }\end{array}$ & $\begin{array}{l}\text { CCL2, CCL5 and CXCL12 recruit macrophages to hypoxic tumor regions and trap them } \\
\text { in TME }\end{array}$ & $\begin{array}{l}{[23,} \\
24]\end{array}$ \\
\hline & Metabolites & $\alpha-K G$ & $\begin{array}{l}\text { - } \alpha-K G \text { induces M2 polarization by promoting the Jmjd3-dependent demethylation } \\
\text { of H3K27 } \\
\text { - } \alpha-K G / \text { succinate ratio determines macrophage polarization status, and higher } \\
\text { ratio presents the } M 2 \text { polarization tendency }\end{array}$ & {$[25]$} \\
\hline & & Adenosine & $\begin{array}{l}\text { Adenosine binds to ADORA, therein, ADORA } 2 A \text { shows dominant M2 polarization } \\
\text { function }\end{array}$ & $\begin{array}{l}{[26,} \\
27]\end{array}$ \\
\hline & & Lactate & $\begin{array}{l}\text { - } \quad \text { Macrophages can sense lactate through Gpr132 and undergo M2 polarization } \\
\text { - } \quad \text { Downregulation of Gpr132 can improve breast cancer metastasis-free survival }\end{array}$ & $\begin{array}{l}{[28,} \\
29]\end{array}$ \\
\hline \multirow[t]{13}{*}{ DCs } & Factors & VEGF & - VEGF inhibits DC differentiation and maturation & {$[80]$} \\
\hline & & IL-10 & IL-10 inhibits DC maturation, activation, and the T cell stimulatory abilities of DCs & $\begin{array}{l}{[82,} \\
83]\end{array}$ \\
\hline & & IL-6, TGF- $\beta$, CSF1 & $\begin{array}{l}\text { - IL-6, TGF- } \beta \text { and CSF1 work as negative modulators of DC maturation and } \\
\text { activation }\end{array}$ & [78] \\
\hline & & & - CSF1 induces the DCs towards suppressive TADCs via Jak2/STAT3 signaling & [84] \\
\hline & & PSA & PSA co-cultural DCs exhibit the impaired mutation phenotypes & {$[85]$} \\
\hline & & MUC1 & MUC1 is related with DC immaturation and anti-tumor function decrease & $\begin{array}{l}{[86,} \\
87]\end{array}$ \\
\hline & Metabolites & Lactic acid & Lactic acid can suppress DC activation & [89] \\
\hline & & IDO & IDO drives DCs toward an immunosuppressive phenotype & [90] \\
\hline & & Extracellular & - Extracellular lipids can be imported and accumulate within DCs & [91] \\
\hline & & lipids & - $\quad$ High lipid content affects the DCs antigen presentation function & [92] \\
\hline & & Wnt5 & $\begin{array}{l}\text { Wnt5a-induced FAO stimulates IDO, thus creating an immunosuppressive } \\
\text { environment }\end{array}$ & [93] \\
\hline & & PGE2 & PGE2 contributes to DC dysfunction & [96] \\
\hline & & $\operatorname{cox}$ & COX impairs the accumulation of $\mathrm{CDC} 1 \mathrm{~s}$ within tumors and suppresses their activation & [97] \\
\hline \multirow[t]{2}{*}{ Neutrophils } & Factors & G-CSF & G-CSF plays an important role in inducing immunosuppressive neutrophils & [133] \\
\hline & & TGF- $\beta$ & TGF- $\beta$ leads to the presentation of a pro-tumor TAN population & [129] \\
\hline
\end{tabular}

CSF1, colony stimulating factor 1; HMGB1, high mobility group box-1; OSM, oncostatin M; CCL, C-C chemokine ligand; CXCL, CXC chemokine ligand; HIF-1 $\alpha$, hypoxia-inducible factors $1 \alpha ; \alpha-K G, \alpha$-ketoglutarate; ADORA, adenosine receptors; Gpr132, G-protein-coupled receptor 132; VEGF, vascular endothelial growth factor; TGF- $\beta$, transforming growth factor- $\beta$; PSA, prostate-specific antigen; MUC1, mucin 1 ; IDO, indoleamine 2,3-dioxgenase-1; PGE2, prostaglandin E2; COX, cyclooxygenase; FAO, fatty acid oxidation; G-CSF, granulocyte colony-stimulating factor.

\section{TAMs promote tumor cell invasion}

Metastasis initiates when tumor cells acquire invasive activity and are able to escape from the confines of the basement membrane into the surrounding stroma. Highly invasive tumor cells always share the characteristics of a loss of intrinsic polarity and loose attachments to surrounding tissue structures [32]. Epithelial-mesenchymal transition (EMT) is a central event in this morphological transformation process, and it contributes to malignant biological properties including invasion and metastasis [33]. During the EMT process, tumor cells lose their cell-cell junctions and apicalbasal polarity as a result of E-cadherin repression, leading to the acquisition of a motile mesenchymal cell phenotype [33]. Recent evidence suggests that TAMs are involved in the regulation of the EMT in various cancer types [34-36]. For example, after co-culture with TAMs, the expression of epithelial marker E-cadherin was reduced in CRC cells, while the mesenchymal marker Vimentin was upregulated, suggesting that TAMs can induce the CRC cell EMT in vitro. This research also revealed that TAMs-derived IL-6 induce EMT by regulating adenylate kinase 2 (AK2)/signal transducer 
and activator of transcription 3 (STAT3)/miR-506-3p/ Forkhead box Q1 (FoxQ1) axis [34]. Biologically, TAMs secrete a series of inflammatory factors, such as IL-6, IL-10, tumor necrosis factor- $\alpha$ (TNF- $\alpha$ ), and transforming growth factor- $\beta$ (TGF- $\beta$ ), thereby promoting EMT [37].

The extracellular matrix (ECM) provides both the biochemical and biomechanical context within which tumor cells exist. Cancer progression is dependent on the ability of tumor cells to traverse the ECM barrier, access systemic circulation, and establish distal metastases. TAMs support tumor cell migration, invasion, and metastasis via ECM remodeling, secreting a number of proteolytic enzymes including cathepsins (B, S, C, L, Z), lysosomal enzymes, matrix metalloproteinases (MMPs, including MMP1, MMP9, MMP12, and MMP14), serine proteases, and disintegrin and metalloproteinase domain-containing protein 10 (ADAM10), which are important components mediating ECM degradation and cell-ECM interactions [38]. In another study, TAMs isolated from breast cancers were found to secrete CCL18, which signals via the breast tumor cell-specific membrane-associated phosphatidylinositol transfer protein 3 (PITPNM3) receptor. This signaling cascade activates integrin clustering on tumor cells, promoting integrin-ECM interactions and adhesion, and thereby facilitating invasiveness and metastasis [39].

In addition to TAMs, some TRMs also present their functions in cancer metastasis. For example, pulmonary alveolar macrophages prepare the appropriate microenvironment for promoting breast cancer metastasis to the lung. They accumulate in the premetastatic lungs through complement $\mathrm{C} 5 \mathrm{a}$ receptor-mediated proliferation and alter the ratio the Th1/Th2 cells, suppressing tumoricidal Th1 and promoting Th2 generation [40]. Kupper cells are TRMs in the liver, which can rely on Dectin-2 to eliminate cancer cells then to inhibit liver metastasis, whereas bone marrow-derived macrophages cannot [41].

\section{TAMs promote tumor cell angiogenesis}

A few studies have shown that the levels of TAMs are closely associated with the vascularization of tumors. Macrophage infiltration into the nonmalignant primary tumors is followed by the formation of a vessel network and inhibition of the Response to suggestion macrophage infiltration can delay this process [42]. In addition to affecting the formation of new tumor vessels, TAMs also stimulate the remodeling of the established vasculature towards a more tortuous and leaky form that favors tumor dissemination [43]. Previous analyses have established the critical role of TAMs-derived VEGF and MMP9 in the promotion of tumor angiogenesis [44]. In addition, TAMs also release a panel of pro-angiogenic factors that include TNF- $\alpha$, basic fibroblast growth factor (bFGF), thymidine phosphorylase (TP), urokinase-type plasminogen activator ( $\mathrm{UPA}$ ), adrenomedullin (ADM), and semaphorin 4D (Sema4D) [45]. There is a recently identified novel subset of TAMs expressing tyrosine-protein kinase receptor 2 (Tie-2) referred to as Tie2-expressing monocytes (TEMs) [46]. TEMs are attracted into tumors by endothelial cell (EC)-derived cytokine angiopoietin-2 (ANG-2), which interacts with its receptor Tie-2 [47]. A growing body of evidence indicates that TEMs in mice and humans significantly contribute to tumor angiogenesis [48]. Selective elimination of TEMs by TEMs specific makers on the surface may thus represent another promising approach to preventing angiogenesis and tumor progression. In addition, given the overlapping roles of TAM-derived VEGF-A and MMP9 in angiogenesis and lymphangiogenesis, this may represent a series of mechanisms whereby macrophages can stimulate lymphangiogenesis. Future studies will shed light on the role of TAMs in the complex control of tumor lymphangiogenesis via the production of cocktails of regulatory cytokines and other factors associated with this process [45].

\section{Regulation of other cells in TME by TAMs}

TAMs play a specific functional role in the context of immune suppression by interaction with $\mathrm{T}$ cells (Figure 1B). Cytotoxic T lymphocyte (CTL) are the major killers of tumor cells [49], while TAMs directly inhibit CTLs responses through the expression of programmed death-protein ligands 1 (PD-L1) on their surfaces, by producing inhibitory cytokines (such as IL-10 and TGF$\beta$ ), and by depleting L-arginine via expression of inducible nitric oxide synthase (iNOS) or arginase I, which results in reactive oxygen species (ROS) production [23]. Tregs are specialized in suppressing anti-tumor immune responses and their infiltration into tumor tissues is often associated with poor prognosis [50]. TAM-derived prostaglandin E2 (PGE2) and IL-10 can promote the induction of Tregs, meanwhile, TAM-derived CCL17/18/22 can recruit additional Tregs, further resulting in CTL suppression [31]. In addition, macrophage production of IL-10 suppresses IL-12 expression by DCs in breast cancer, limiting cytotoxic $\mathrm{CD}^{+} \mathrm{T}$ cell responses during chemotherapy (Figure 1B) [51]. In pancreatic ductal adenocarcinoma, IL-33-induced TAMs-CXCL3 production targets CXC-chemokine receptor 2 (CXCR2) on fibrotic cells to cause a fibroblast-to-myofibroblast transition which associates with an increased risk of cancer metastasis [52]. 


\section{Therapeutic targeting of TAMs}

\section{Targeting TAMs recruitment and activation}

As discussed above, a majority of TAMs originate from bone marrow monocyte procurers. Recruitment of TAMs to the tumor sites is a consequence of the continuous presence of tumor-derived chemoattractants. As such, cutting off those signals may represent a promising approach to modulating TAMs responses to suppress tumor growth (Table 2). For example, CSF1 and its receptor, CSF1R, regulate the migration, differentiation, and survival of macrophages and their precursors [53]. This CSF1/CSF1R axis has been heavily investigated in preclinical models and clinical trials. In a phase 1 study, anti-CSF1R therapy exhibited an ability to efficiently deplete TAMs in cancer patients and to promote a switch from infiltration primarily by $\mathrm{CD}^{+} \mathrm{T}$ cells towards infiltration primarily by $\mathrm{CD}^{+} \mathrm{T}$ cells [54]. Additionally, CSF1/CSF1R blockade improves the efficacy of a diverse range of immunotherapeutic treatment regimens, including CD40 agonists, PD-1 or CTLA- 4 antagonists, and adoptive $\mathrm{T}$ cell therapy. These findings have spurred the development of several clinical trials combining CSF1 and/ or CSF1R inhibitors with immune checkpoint blockade agents. In one promising study of patients with pancreatic cancer, who did not traditionally respond to immunotherapy, researchers found that some patients responded to a combination of CSF1R and PD-1 antagonists [55], and these studies are now moving forward towards a multi-arm phase II clinical trial. However, the efficacy of CSF1R-based therapies remains somewhat controversial. According to a single-cell analysis, such CSF1R blockade exhibits a cell cycle preference and is thus insufficient to deplete all macrophage populations [12].

Table 2: Therapeutic strategies targeting tumor-associated macrophages, DCs, and neutrophils.

\begin{tabular}{|c|c|c|c|c|c|c|}
\hline Effects & $\begin{array}{l}\text { Class of } \\
\text { agents }\end{array}$ & Agents & Combination & Cancer type & Phase & Trial ID \\
\hline \multirow[t]{9}{*}{$\begin{array}{l}\text { Targeting TAM recruitment } \\
\text { and activation }\end{array}$} & \multirow[t]{8}{*}{$\begin{array}{l}\text { CSF1R } \\
\text { inhibitors }\end{array}$} & Pexidartinib & $\begin{array}{l}\text { Durvalumab (PD-L1 } \\
\text { antagonists) }\end{array}$ & $\begin{array}{l}\text { Metastatic/advanced } \\
\text { pancreatic or colorectal } \\
\text { cancers }\end{array}$ & Phase 1 & NCT02777710 \\
\hline & & LY3022855 & $\begin{array}{l}\text { Durvalumab or } \\
\text { tremelimumab }\end{array}$ & Advanced solid tumors & Phase 1 & NCT02718911 \\
\hline & & IMC-CS4 & Monotherapy & Advanced solid tumors & Phase 1 & NCT01346358 \\
\hline & & Cabiralizumab & $\begin{array}{l}\text { Nivolumab (PD-1 } \\
\text { antagonists) }\end{array}$ & Selected advanced cancers & Phase 1 & NCT02526017 \\
\hline & & Cabiralizumab & Monotherapy & $\begin{array}{l}\text { Diffuse type tenosynovial } \\
\text { giant cell tumor }\end{array}$ & $\begin{array}{l}\text { Phase } \\
1 / 2\end{array}$ & NCT02471716 \\
\hline & & Pexidartinib & Monotherapy & $\begin{array}{l}\text { Giant cell tumor of the } \\
\text { tendon sheath }\end{array}$ & Phase 3 & NCT02371369 \\
\hline & & ARRY-382 & $\begin{array}{l}\text { Pembrolizumab (PD-1 } \\
\text { antagonists) }\end{array}$ & Advanced solid tumors & Phase 2 & NCT02880371 \\
\hline & & ARRY-382 & Monotherapy & $\begin{array}{l}\text { Selected advanced or meta- } \\
\text { static cancers }\end{array}$ & Phase 1 & NCT01316822 \\
\hline & $\begin{array}{l}\text { CCR2 } \\
\text { antagonists }\end{array}$ & MLN1202 & Monotherapy & Bone metastases & Phase 2 & NCT01015560 \\
\hline \multirow{7}{*}{$\begin{array}{l}\text { Targeting TAM reprogram- } \\
\text { ming and DC activation }\end{array}$} & \multirow[t]{7}{*}{ CD40 agonists } & APX005M & Monotherapy & Solid tumors & Phase 1 & NCT02482168 \\
\hline & & APX005M & $\begin{array}{l}\text { Nivolumab (PD-1 } \\
\text { antagonists) }\end{array}$ & $\begin{array}{l}\text { Non-small cell lung cancer } \\
\text { or metastatic melanoma }\end{array}$ & $\begin{array}{l}\text { Phase } \\
1 / 2\end{array}$ & NCT03123783 \\
\hline & & CP-870,893 & $\begin{array}{l}\text { Tremelimumab (CTLA4 } \\
\text { antagonists) }\end{array}$ & Metastatic melanoma & Phase 1 & NCT01103635 \\
\hline & & ChiLob 7/4 & Monotherapy & $\begin{array}{l}\text { Advanced malignancies } \\
\text { refractory }\end{array}$ & Phase 1 & NCT01561911 \\
\hline & & Selicrelumab & $\begin{array}{l}\text { Atezolizumab (PD-L1 } \\
\text { antagonists) }\end{array}$ & $\begin{array}{l}\text { Locally advanced and/or } \\
\text { metastatic solid tumors }\end{array}$ & Phase 1 & NCT02304393 \\
\hline & & HCD122 & Monotherapy & Multiple myeloma & Phase 1 & NCT00231166 \\
\hline & & Selicrelumab & $\begin{array}{l}\text { Vanucizumab or } \\
\text { bevacizumab }\end{array}$ & Metastatic solid tumors & Phase 1 & NCT02665416 \\
\hline \multirow[t]{2}{*}{$\begin{array}{l}\text { Targeting neutrophil } \\
\text { infiltration }\end{array}$} & \multirow[t]{2}{*}{$\begin{array}{l}\text { CXCR2 } \\
\text { antagonists }\end{array}$} & Reparixin & Paclitaxel & $\begin{array}{l}\text { Metastatic triple-negative } \\
\text { breast cancer }\end{array}$ & Phase 2 & NCT02370238 \\
\hline & & Reparixin & Paclitaxel & $\begin{array}{l}\text { HER2 negative metastatic } \\
\text { breast cancer }\end{array}$ & Phase 1 & NCT02001974 \\
\hline
\end{tabular}


C-C chemokine receptor 2 (CCR2) inhibition leads to monocyte retention within the bone marrow, resulting in a depleted pool of circulating cells and reduced numbers of TAMs in primary and metastatic sites [56]. In preclinical models, CCL2 or CCR2 blockade can improve the efficacy of chemotherapy, radiotherapy, and immunotherapy. Several CCR2 blockade combination clinical trials are therefore ongoing. The CCR2 inhibitors PF-04136309 and CCX782 both show safety and efficacy in patients with pancreatic cancer. When combined with FOLFIRINOX, a greater than $40 \%$ increase in responsiveness to chemotherapy has been observed together with the prolongation of patient overall survival $[57,58]$. Biomarker analyses have also suggested that combination therapy was associated with increased $\mathrm{CD}^{+}$and $\mathrm{CD}^{+}{ }^{+} \mathrm{T}$ cell infiltration together with reductions in levels of Tregs [57]. Similar observations have also been made in some preclinical models, facilitating further combinations with checkpoint immunotherapy. Clinical testing of this triple combination of CCR2 inhibition, chemotherapy, and checkpoint blockade is now ongoing. Although CCR2 inhibitors play a promising role in therapy, they remain subject to significant shortcomings. Indeed, the cessation of CCL2 and/or CCR2 blockade can lead to a release of the monocytes previously trapped within the bone marrow, and this has been shown to exacerbate metastasis in a murine model of breast cancer [59].

\section{Targeting TAM reprogramming}

As discussed above, one of the key features of macrophages is their plasticity, which enables them to change their phenotype in the tumor microenvironment. Reprogramming TAMs to an anti-tumor phenotype thus represents an attractive therapeutic strategy. One of the most effective approaches to such reprogramming identified to date has been the use of a CD40 agonist antibody. CD40-activated macrophages are indicative of M1 phenotype correlating with enhanced pro-inflammatory cytokine production [60]. In mouse model, CD40 pathway can be harnessed to restore tumor immune surveillance by targeting TAMs [61]. As CD40 is mainly expressed by classical dendritic cells (cDCs), the relative contribution of TAMs and $\mathrm{CDC}$ activation is unclear. However, enhanced responses to PD-1 and CTLA- 4 antagonists have been observed following such treatment [62]. $\beta$-glucan, a yeast-derived polysaccharide, has also been shown to promote TAM differentiation into an M1 phenotype and is a potent immunomodulator with anti-cancer properties [63].

\section{Potential therapies targeting TAMs}

Tumor-expressed CD24 could interact with inhibitory receptor sialic-acid-binding Ig-like lectin 10 (Siglec-10) on TAMs to promote immune evasion were validated in ovarian cancer and breast cancer [64]. Research showed that blockade or knocking out Siglec-10 augmented the phagocytic ability of macrophages, which demonstrated the potential of Siglec-10 as immune checkpoint in immunotherapy [64]. In addition, there is increasing attention to the unique subset of macrophages expressing the cell surface receptor TREM2, which are present in different types of tumors $[65,66]$. Lack of TREM2 influences the macrophage subsets proportion, diminishing the immunosuppressive clusters while the clusters which express immunostimulant gene increase [67]. Meanwhile, anti-TREM2 treatment curb tumor growth in mice and enhance the anti-PD-1 efficiency, showing its potential in combination therapy [67]. Emerging technological advances, such as single-cell RNA sequencing (scRNA-seq) and spatial transcriptomics technology that preserves spatial information, will further accelerate the search for novel potential targets on TAMs.

Tumor cells can regulate TAMs polarization, both through tumor-derived factors and metabolites. In turn, TAMs can promote tumor progression. This crosstalk between TAMs and tumor cells makes TAMs as one of the most essential pro-tumor cells and treatment strategies targeting TAMs are anticipated to be feasible.

\section{Dendritic cells}

DCs are central mediators of the initiation and regulation of anti-tumor immunity. DCs migrate to the tumor sites, internalize portions of moribund tumor cells, and respond to stimuli that can drive DC maturation, emigration from the tumor site, and homing to regional lymph nodes where they can present tumor-derived antigens to antigenspecific T cells. Activated T cells upregulate the expression of chemokine receptors, enabling circulating CTLs to infiltrate the tumor and to destroy malignant cells [60]. Here, we review the DCs phenotypes driven by interactions with the TME and with T cells. Therapies targeting these interactions are also discussed.

\section{DC subset functions}

DCs are professional antigen-presenting cells that prime effector $\mathrm{CD}^{+}$or $\mathrm{CD}^{+} \mathrm{T}$ cell responses [68]. Previous work on the development of DCs from bone marrow progenitors 
identified a population named as the common dendritic cell precursors (CDPs), which give rise to plasmacytoid DCs progenitors (pre-pDCs) and classical DCs progenitors (precDCs) [69]. pDCs and cDCs are the most common and beststudied DC subsets (Figure 2A) [70], with cDCs being further separated into $\mathrm{CD}_{103^{+}} \mathrm{cDC} 1$ and $\mathrm{CD} 11 \mathrm{~b}^{+} \mathrm{cDC} 2$ subtypes based on their phenotypic and functional characteristics. cDC1s support stronger $\mathrm{CD}^{+} \mathrm{T}$ cell immunity and induce the Th1 cells polarization of $\mathrm{CD}^{+} \mathrm{T}$ cells through the secretion of IL-12 [71, 72]. Th1 cells are responsible for cellmediated immunity and phagocyte-dependent protective responses [73]. While, cDC2s appear to be essential for the priming of anti-tumor $\mathrm{CD}^{+}{ }^{+} \mathrm{T}$ cell responses [74]. In addition, inflammatory conditions can lead to the formation of monocyte-derived DCs (moDCs), which play an essential role in defenses against pathogens by participating in the induction of both adaptive and innate immune responses [75]. Advancements in high-throughput single-cell analysis technologies have enabled the identification of novel subsets of dendritic cells in human cancers. For example, lysosomal associated membrane protein $3(L A M P 3)^{+} \mathrm{cDCs}$ have been identified via scRNA-seq data of most cancer types, wherein they exhibit an enhanced migratory capacity and the potential to develop from both cDC1s and cDC2s [16]. Owing to the heterogeneity of $\mathrm{CDC} 2$ cell populations, T-bet and RAR-related orphan receptor gamma (RORyt) expression can be assessed to define distinct CDC2 subsets, including anti-inflammatory $\mathrm{T}^{\mathrm{T}}$ bet $^{+} \mathrm{CDC} 2 \mathrm{~A}$ and proinflammatory T-bet ${ }^{-}$cDC2B cells [76]. Single-cell analyses have extended current knowledge of DC heterogeneity, but further such studies are needed to dissect their functional roles in tumors and related therapeutic contexts.

\section{Tumor microenvironmental remodeling of DC characteristics}

Tumors can evolve multiple mechanisms that enable them to thrive under adverse conditions while actively suppressing the protective function of immune cells. Previous studies have reported that DC tolerization is involved in tumor-mediated immune evasion [77]. Herein, we discuss the extrinsic mediators and metabolic program changes in TME that can influence DC functionality.

\section{Extrinsic mediators}

Several mediators present in the tumor site can directly alter the activaity of infiltrating DCs to promote malignant progression, including secreted proteins (growth factors, cytokines, chemokines), tumor antigens, and other factors (Figure 2A and Table 1) [78].

VEGF is a secreted heparin-binding protein produced by a majority of tumors that is responsible for angiogenic activity [79]. VEGF inhibits the differentiation and maturation of DCs via binding and activating the tyrosine kinase receptors, VEGFR-1 and VEGFR-2 [80]. VEGF is also known to regulate $\mathrm{DC}$ migration and homing by recruiting immature myeloid cells from the bone marrow to the primary tumor site, thereby generating a population of immature DCs [81]. IL-10 is an anti-inflammatory cytokine produced by tumor cells, macrophages, regulatory T cells, and other stromal components. Tumor-derived IL-10 has been shown to inhibit DC maturation, activation, and the T cell stimulatory abilities of DCs $[82,83]$. In addition, IL- 6 , TGF- $\beta$, and CSF1 also play suppressive roles as modulators of DC maturation and activation [78]. Studies show that tumorderived factors in the TME, especially CSF1, induce the development of immature, tolerogenic tumor-associated DCs (TADCs) that contribute to tumor progression via Janus kinase 2 (Jak2)/STAT3 signaling [84].

Tumor antigens can also potentially function as DC-suppressive factors, For example, prostate-specific antigen (PSA), which is a serine protease overexpressed in most prostate cancers, was the first tumor-associated antigen shown to inhibit DC maturation, longevity, and function [85]. DCs cultured in the presence of active PSA exhibited significantly reduced CD83, CD80, CD86, and human leukocyte antigen (HLA)-DR expression consistent with impaired maturation. Mucins such as MUC1, a glycoprotein overexpressed in many tumor cells, are responsible for impaired DC maturation and function [86]. When cultured in the presence of MUC1, immature DCs exhibited CD83, CD80, CD86, and CD40 upregulation together with the production of higher levels of IL- 6 , TNF- $\alpha$, and IL-10, but they fail to produce IL-12, and thus do not induce Th1 cells responses which are crucial to anti-tumor immunity [87].

\section{Metabolic reprogramming}

The unique features of the TME including hypoxia, scarce nutrient availability, and competition for amino acids can induce metabolic perturbations within TADCs [88], thereby interfering with the development of robust anti-tumor immune responses. For example, tumor-derived lactic acid can suppress DC activation in vitro, and blocking of lactic acid production reverts the TADCs phenotype to normal [89]. The metabolism of tryptophan by indoleamine 2,3-dioxygenase 
A

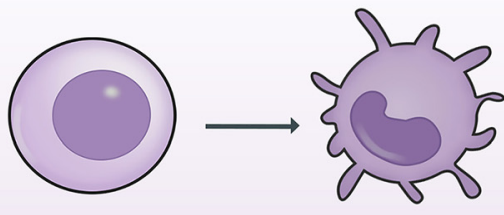

Pre-DCs
Immature DCs
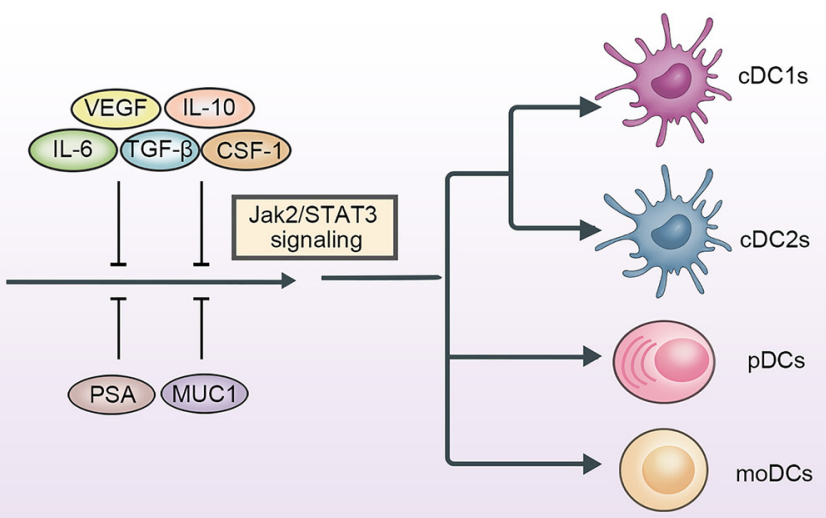

Mature DCs

B

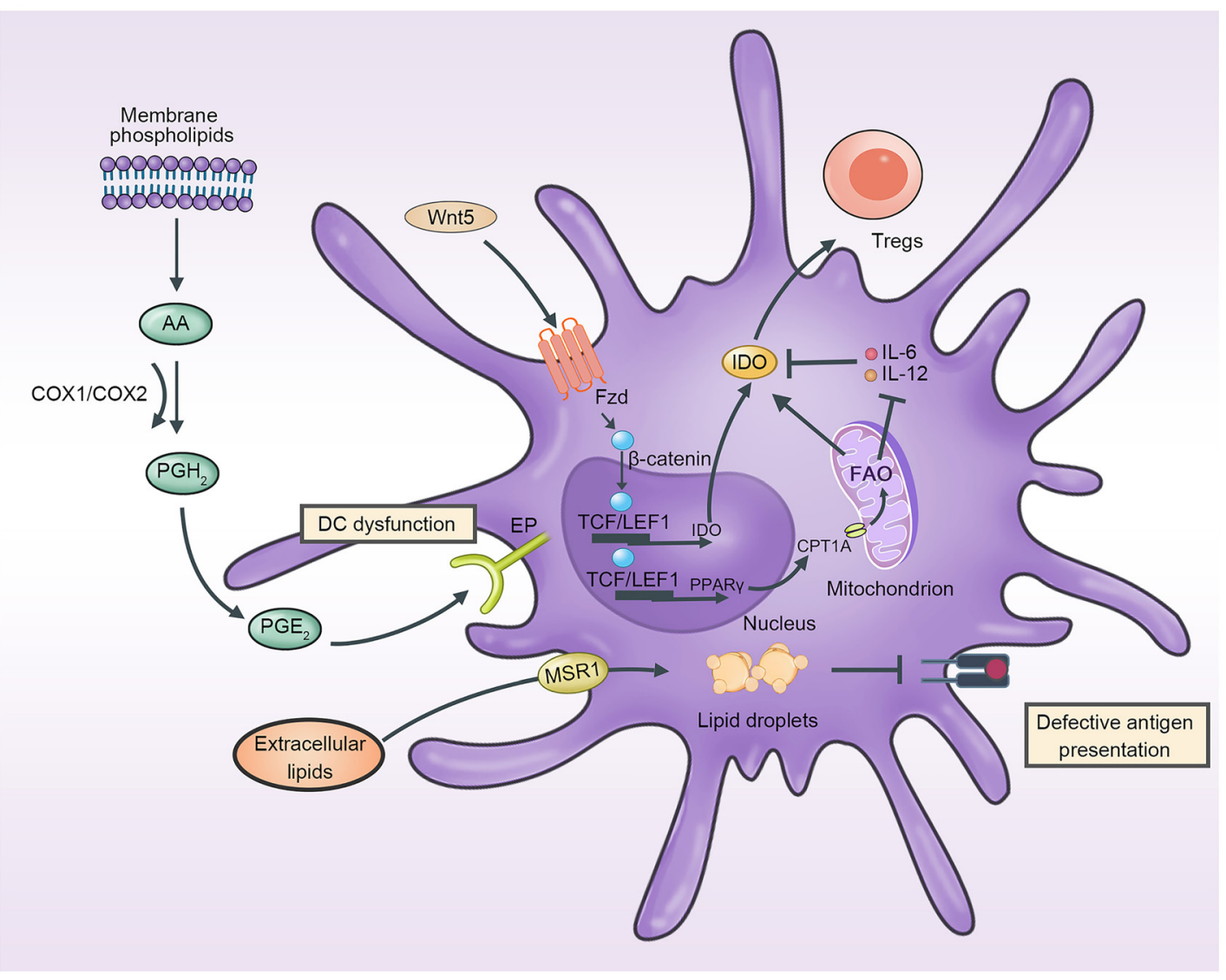

Figure 2: DCs origin and reprogramming in TME.

(A) Tumor cells influence on DCs development. $C D C 1 s, c D C 2 s, p D C s$ and moDCs are predominant $D C s$ subsets. They present various phenotypes and functions. Tumor-derived factors (VEGF, IL-10, IL-6, TGF- $\beta$, and CSF-1) and tumor antigens (PSA, MUC1) interfere the DCS maturation. (B) Lipid-mediated DCs metabolic reprogramming. DCs lipid metabolism and bioactive lipid sensing can drive tolerogenic TADC polarization. High lipid content defect DCs antigen presentation function. Tumor-derived Wnt5a triggers DC FAO via a $\beta$-catenin-PPAR- $\gamma$ pathway, thereby supporting IDO enzymatic activity and subsequent Tregs differentiation. PEG2 leads to DC dysfunction, suppressing the differentiation of Th1-inducing DCs. pDCs, plasmacytoid DCs; cDCs, classical DCs; moDCs, monocyte-derived DCs; VEGF, vascular endothelial growth factor; TGF- $\beta$, transforming growth factor- $\beta$; CSF-1, colony stimulating factor 1; PSA, prostate-specific antigen; MUC1, mucin 1; AA, arachidonic acid; COX, cyclooxygenase; PGH2, prostaglandin H2; PGE2, prostaglandin E2; EP, E-prostanoid receptors; FZD, frizzled; FAO, fatty acid oxidation; IDO, indoleamine 2,3-dioxgenase-1; MSR1, macrophage scavenger receptor 1. 
(IDO) in the TME can drive the generation of Tregs, and thereby inhibiting T cell-mediated anti-tumor immunity [90].

Lipid metabolism plays a particularly important regulatory role in this context (Figure 2B). Owing to tumorrelated metabolic disturbances, lipids in lipid droplets (LDs) can accumulate within cancer cells, allowing survival in a microenvironment with high energy demands [91]. Extracellular lipids can be imported and accumulate within the intracellular space by increased uptake of extracellular lipids due to the upregulation of the macrophage scavenger receptor 1 (MSR1), and DCs with high lipid content have defects in processing of tumor-associated proteins [92]. A recent study indicated that tumor-derived Wnt5a can induce $\beta$-catenin-peroxisome proliferator-activated receptor-y (PPARy) signaling in TADCs, thus shifting DC metabolic activity away from glycolysis and towards fatty acid oxidation (FAO). This metabolic program effectively inhibits the activation of effector $\mathrm{T}$ cells while driving the differentiation of Tregs [93]. Furthermore, Wnt5a-induced FAO plays a critical role in regulating DC metabolism, as it suppresses IL- 6 and IL-12 expression, in addition to stimulating IDO enzymatic activity, creating an environment conducive to the generation of Treg cells [93]. Blocking FAO in combination with anti-PD-1 treatment may represent an effective therapeutic strategy. Notably, bioactive lipids, such as PGE2, have been shown to function as key signaling molecules that modulate TADC function. Membrane lipids can be metabolized to generate PGE2 via the cyclooxygenase-2 (COX)/PGE2 pathway [94, 95]. Notably, tumor-derived PGE2 can disrupt the early stages of DC differentiation, contributing to DC dysfunction in cancer [96]. Tumor-derived COX also impairs the accumulation of cDC1s within tumors and suppresses their activation, including IL-12 production. The implantation of COX-deficient transplantable tumors in basic leucine zipper transcription factor ATF-like 3 (Batf3)-knockout mice lacking cDC1s does not lead to impaired tumor growth [97]. Overall, current evidence indicated that intracellular lipid metabolism and bioactive lipid sensing in the TME can effectively drive tolerogenic TADC polarization.

\section{Signals involved in the interplay between $D C s$ and $T$ cell}

In the TME and tumor-draining lymph nodes (TDLNs), DCs present tumor-associated antigens to $\mathrm{T}$ cells, thereby promoting antigen-specific $\mathrm{T}$ cell activation and proliferation. However, such antigen presentation alone is insufficient to prime effective anti-tumor immunity. Many other signals also influence these interactions between DCs and T cells, including costimulatory molecules, cytokines, and chemokines (Figure 3).

The expression of CD80 and CD86 on DCs can control the activation or suppression of $\mathrm{T}$ cells through interactions with CD28 and CTLA-4, respectively [98]. TADCs express a high level of PD-L1 and the expression is upregulated during antigen-presentation to protect DCs from cytotoxicity of activated T cells. However, DC-derived PD-L1 suppress T cell activation and cytokine production, thus dampening the anti-tumor immune responses [99]. Besides, there are many DCs costimulatory molecules working on anti-tumor effect. CD40 on DCs has been shown to interact with CD40L on $\mathrm{T}$ cells, enhancing $\mathrm{T}$ cell stimulatory capacity and favoring Th1 cells responses [100]. Th1-driving effector DC (DC1) is a special DCs subtype which expresses elevated levels of intercellular adhesion molecule 1 (ICAM-1) but produces only low levels of IL-12, excluding the influence of IL-12 on Th1 polarization [101]. ICAM-1-expressing DC1 drive Th1 polarization, and blocking ICAM-1/LFA-1 interactions in cocultures of DCs and naïve T cells can attenuate Th1 polarization [101]. Similarly, DCs that express OX40L give rise to a primary Th1 cells response and vaccination of such $0 X 40 \mathrm{~L}^{+}$ DCs results in significant enhancement of therapeutic antitumor effect [102]. Moreover, the expression of both CD70 (CD27L) and glucocorticoid-induced TNFR-related protein (GITRL) GITRL on DCs can support $\mathrm{CD}^{+} \mathrm{T}$ cell priming, thereby inducing anti-tumor immunity [103].

DC-derived cytokines can promote the development of either a pro-inflammatory or anti-inflammatory microenvironment. The effector activity of $\mathrm{T}$ cells is dependent upon DC-derived cytokines, including IL-12 and type I interferons (IFN- $\alpha / \beta)$, which are pro-inflammatory factors that shape Th1 cells development $[104,105]$. IL-12 production by DCs requires both CD40L and IFN-y signals [106]. Type I interferons can inhibit IFN-y signaling, thereby restricting IL-12 expression by DCs, suggesting that these two signaling pathways can antagonize one another [107]. It has also been shown that DC-derived IL-10 and TGF- $\beta 1$ are associated with the initiation and progression of cancer [108]. They can support Treg development and serve as anti-inflammatory mediators $[109,110]$.

DCs can also produce chemokines in the TME that attract T cells. CXCL9 and CXCL10, which bind to CXCR3, are key chemokines necessary for the intratumoral trafficking of effector T cells [111]. It is important to note that cDC1s serve as the primary source of CXCL9 and CXCL10, and BATF3 deletion or a lack of cDC1s can result in impaired effector T cell trafficking and defective anti-tumor immunity [111]. Moreover, CXCR3 can also be expressed by Treg cells. As such, cDC1s may recruit other immunosuppressive cells into the TME, 


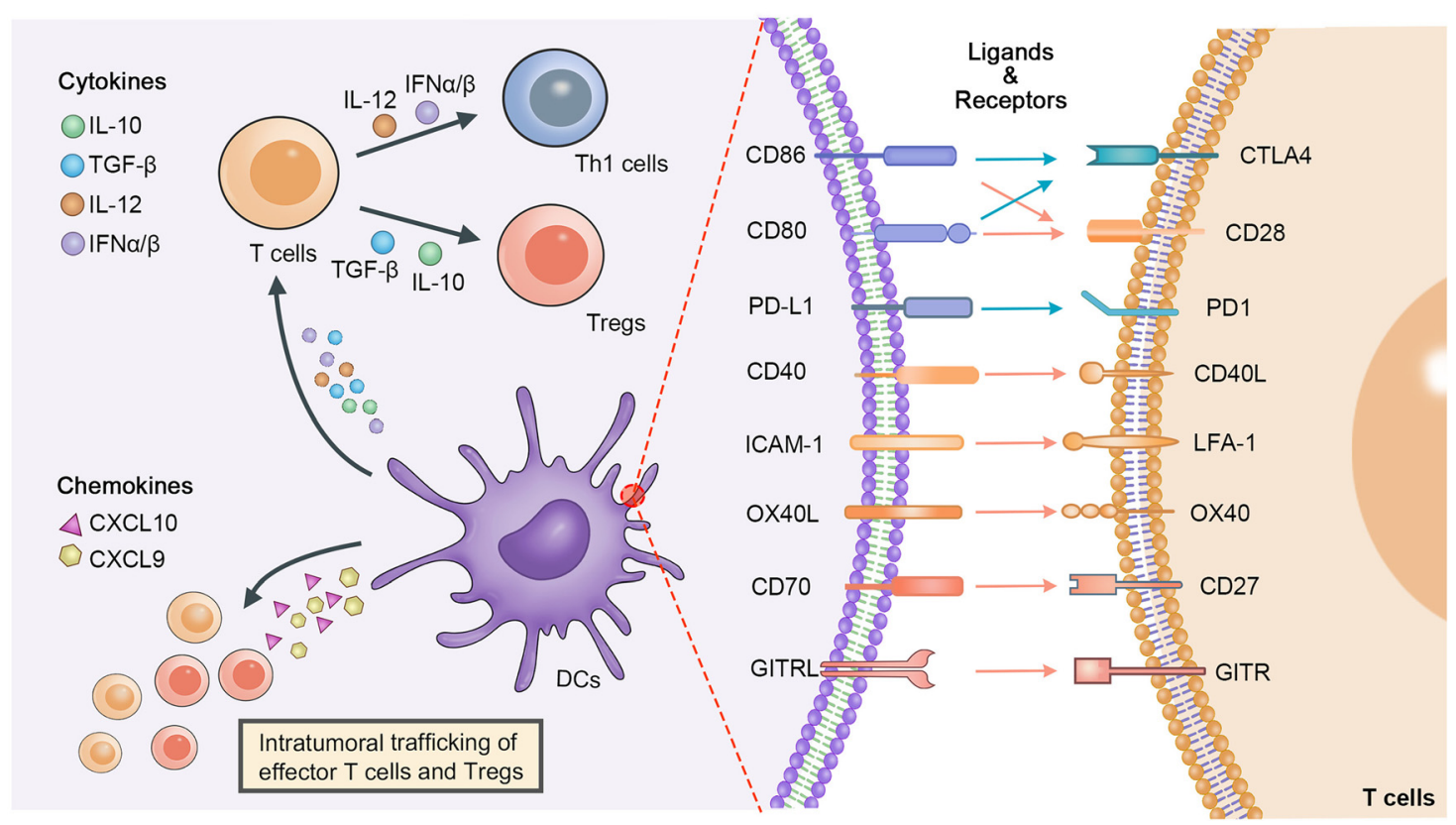

Figure 3: Signals between DCs and T cells.

Costimulatory molecules play contrary roles. Some of them suppress T cell activation (blue arrows and receptors), while others favor Th1, CTL cell responses (orange arrows and receptors). DCs secrete IL-12 and IFN $\alpha / \beta$ to shape Th1 cells development, meanwhile, TGF- $\beta$ and IL-10 can support Treg development. CXCL9/10 are produced by TADCs, binding to CXCR3 on effector T cells and Treg cells and then recruiting them into TME. CTLA-4, cytotoxic T lymphocyte antigen 4; PD-1, programmed cell death protein 1; PD-L1, programmed cell death 1 ligand 1; ICAM-1, intercellular adhesion molecule 1; LFA-1, lymphocyte function-associated antigen 1; GITR, glucocorticoid-induced TNFR-related protein; GITRL, GITR ligand; TGF- $\beta$, transforming growth factor $-\beta$; $C X C L, C X C$ chemokine ligand.

suggesting that the interactions between DCs and T cells may be even more complex than previously recognized. In the context of cancer, TDLN DCs may initially prime naïve, tumor antigen-specific T cells, while DCs in the TME may further license the migration of antigen-primed T cells [112].

\section{DCs-based immunotherapy in cancer treatment}

DC-based immunotherapies are being actively developed, and can be broadly classified into ex vivo and in vivo approaches. Ex vivo approaches include traditional DCs vaccines, which rely on in vitro DC antigen loading, activation, and cytokine treatment prior to injection back into patients [113]. In vivo approaches, in contrast, target DCs within patients in an effort to enhance their antitumor activity. Approaches to enhancing DC activation and mobilization have been discussed in detail in prior reviews [7, 114]. Herein, we will focus on discussing approaches to restoring DC immune functionality through targeting in vivo DCs ligands, blocking inhibitory signals, and interfering with metabolic pathway activation (Table 2).

\section{Ligation-based in vivo DC targeting}

Ligation-based approaches to DC targeting in vivo include the ligation of CD40, and C-type lectin receptors (CLRs). $\mathrm{CD} 40$, a member of the tumor necrosis factor receptor (TNF-R) family, is a surface receptor best known for its ability to initiate multifaceted activation signals in normal B cells and DCs [115]. CD40 agonist antibodies have been used to provide activation signals necessary for DC-derived pro-inflammatory cytokine production and the enhancement of $\mathrm{T}$ cell activation. Moreover, agonistic CD40 antibodies can be combined with chemotherapy, checkpoint inhibitory antibodies, and other immune modulators to alter therapeutic outcomes [116]. A phase I study in combination with gemcitabine in patients with advanced pancreatic cancer found these antibodies to be well-tolerated and to exhibit anti-tumor activity [117]. In a mouse model, CD40 agonistic antibody treatment can overcome resistance to checkpoint blockade treatment and improve survival [62]. Recent single-cell analyses have highlighted changes in immune cell number and function upon anti-CD40 treatment. In MC38 tumor models, the cDC1s population is specifically amplified by such treatment. In addition, anti-CD40 treatment can impact the expansion, migration, and transition of tumor-infiltrating $\mathrm{CD}^{+}$effector 
memory $\mathrm{T}\left(\mathrm{T}_{\mathrm{em}}\right)$ and tissue-resident memory $\mathrm{T}\left(\mathrm{T}_{\mathrm{rm}}\right)$ cells, enhancing the crosstalk of basic helix-loop-helix family member E40 (BHLHE4O) $)^{+}$Th1-like cells and cDC1s [12].

CLRs are also attractive therapeutic targets, as DC subsets are known to express different CLRs that are involved in the recognition and capture of many glycosylated antigens [118]. The CLR protein family consists of DEC205, Mincle, C-type lectin domain family 9 (CLEC9A), and DC-specific intercellular adhesion molecule-3-grabbing non-integrin (DC-SIGN). Different from directly activating DCs like CD40 antibody, CLRs work as targets receptors for antigen delivering. In previous study, researchers have generated human chimeric antibodies specific for CLEC9A and DEC205, with antigens on C-terminus. Those antibodies can specifically deliver antigens to DCs for processing and presentation to $\mathrm{CD}^{+} \mathrm{T}$ and $\mathrm{CD}^{+} \mathrm{T}$ cells, underscoring the potential clinical utility of this strategy [119]. Human studies using antigens targeted to DEC205 have been shown to specifically induce tumor-specific $\mathrm{T}$ cell responses in subsets of patients [120].

\section{Blocking inhibitory signals}

Overcoming the immunosuppressive activities of TADCs represents another approach to enhancing DC function. One advantage of this approach is that it allows for the systemic administration of inhibitors, as opposed to the local administration approach required for many immune agonists. One of the first examples of this approach was the targeting of VEGF, given that its suppressive effects on DCs have been discussed previously. VEGF inhibitors are already in clinical use for the inhibition of angiogenesis, and anti-VEGF antibodies have been shown to enhance the numbers and functionality of DCs in tumor-bearing mouse model systems [121, 122]. VEGF inhibition has also been shown to enhance DC maturation in human patients, suggesting that this may contribute to the efficacy of VEGF inhibitors in clinical settings [123]. Another potent immunosuppressive signal in TME is STAT3, and STAT3 inhibitors, which can promote DC maturation and activation, are currently being evaluated in clinical trials [124, 125].

\section{Metabolic pathway targeting}

The regulation of immunometabolism as a means of enhancing anti-tumor immunity is a growing area of active research in the field of cancer therapy. Interfering with lipid metabolism represents a particularly attractive approach to enhancing TADC-mediated anti-cancer immunity. PGE2 acts as an anti-inflammatory mediator, inhibiting inflammatory chemokine release from activated DCs [126]. Blocking COX enzymes to limit PGE2 production represents a promising immunotherapeutic approach that may enhance antitumor therapy by interfering with lipid metabolic pathways. For example, aspirin, a nonsteroidal anti-inflammatory drug (NSAID) that can block the COX-1/2 pathway, as well as celecoxib, a COX-2 inhibitor, have been found to improve anticancer treatment outcomes when combined with antiPD-1 therapy in a murine model of melanoma [97]. Moreover, IDO inhibition is actively being studied in mice and in clinical trials. The potential combination of IDO inhibitors with DC-based cancer vaccines is also a topic of ongoing study [127]. Inhibiting fatty acid catabolism may also offer therapeutic potential as a means of restoring TADC functionality. Inhibiting FAO using etomoxir has been shown to improve the ability of DCs to induce T cells activation and suppress Treg cell differentiation ex vivo [93]. Furthermore, a combination of FAO inhibition and anti-PD-1 blockade treatment was shown to exhibit significant improvements in host survival driven by enhanced anti-tumor immunity [93].

Generally, DCs are antigen-presenting cells supporting stronger $\mathrm{T}$ cell anti-tumor immunity. However, the complexity of TME interferes DC maturation and antigen presentation, and even remodels DCs into pro-Tregs differentiation phenotype. Immunotherapy can exclude the immunosuppressive signals or directly activate DCs through receptors on their surface, enhancing DC anti-tumor ability.

\section{Tumor-associated neutrophils}

Neutrophils, which originate from myeloid precursors, compose the significant cellular parts of the leukocyte compartment and are the primary responsive cell type associated with innate immune responses [128]. The TME can control neutrophil recruitment, and tumor-associated neutrophils (TANs) can regulate tumor progression and growth.

\section{Neutrophil classification}

The role of neutrophils in cancer has long been a matter of controversy. In 2009, in an effort to mirror the M1/M2 TAM classification model, neutrophils were classified into N1 (anti-tumor neutrophil) and N2 (pro-tumor neutrophil) subsets [129]. This classification model was based on the discovery that differences in neutrophil polarization were evident following TGF- $\beta$ treatment in mouse model systems. However, the utility of such $\mathrm{N} 1 / \mathrm{N} 2$ polarization is limited given that their surface markers, cytokine expression patterns, transcription 
factor regulators, and other hallmarks of activation are largely unknown. Moreover, neutrophils can be divided into high-density neutrophils (HDNs) and low-density neutrophils (LDNs). LDNs can be further separated into immature MDSCs and mature cells that are derived from HDNs in a TGF- $\beta$-dependent manner (Figure 4). LDNs exhibited impaired levels of activity as compared to HDNs with respect to several basic neutrophil functions, and LDNs are tumor permissive, whereas HDNs play an anti-tumor role [130]. Although the neutrophil with high RNAase content that makes it with lower RNA content under the 10X Genomics platform and requires setting low filtering thresholds to allow their detection [131]. Multiple scRNA-seq data on the development of neutrophils and neutrophils from cancer tissues are emerging. For example, six neutrophil subpopulations were found in mouse lung tumors and five subpopulations were found in human lung tumors, and three subpopulations show conserved gene expression within mouse and human neutrophils [132]. In order to further detect the low gene transcripts of neutrophils, scRNA-seq needs to be performed by other platforms (such as BD Rhapsody) to restore a higher proportion of neutrophil gene transcripts, but still lack related scRNA-seq in tumor tissues to distinct neutrophil subpopulations.

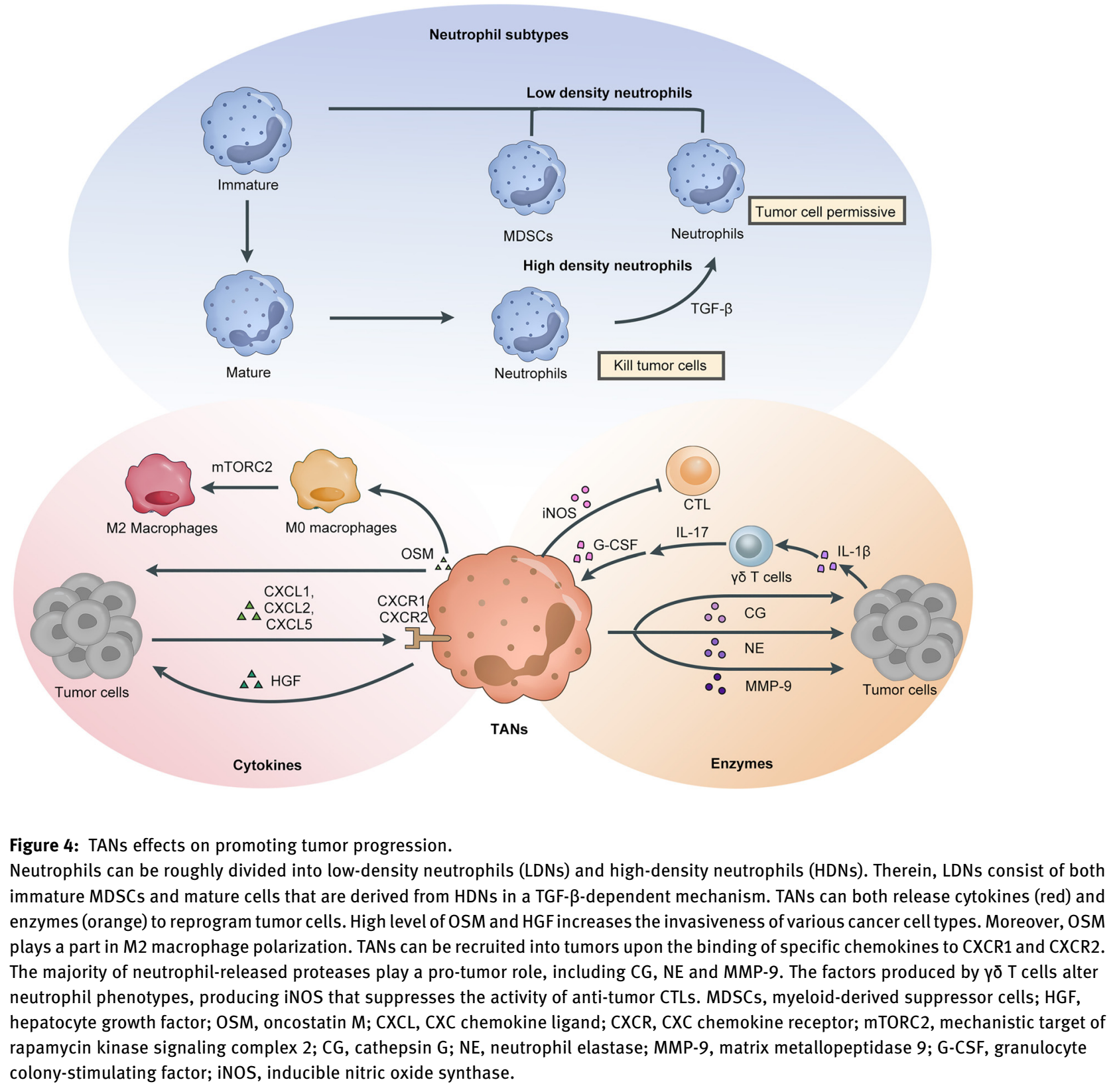




\section{Tumor-derived factors regulating TAN functions}

The phenotypic classification of TANs is partly based on specific tumor-derived factors. Granulocyte-colony stimulating factor (G-CSF) and TGF- $\beta$ are the best-studied molecules in this context (Table 1). G-CSF is the only hematopoietic growth factor that exhibits increased serum expression during early tumor development. Prolonged G-CSF stimulation is both necessary and sufficient to promote the development of tumor-induced immunosuppressive neutrophils [133]. TGF- $\beta$ production within the TME induces a population of TANs with a pro-tumor phenotype. TGF- $\beta$ blockade can increase the production of neutrophil-attracting chemokines, resulting in an influx of TANs that are hypersegmented, more cytotoxic to tumor cells, and express higher levels of proinflammatory cytokines, thereby inducing a more robust anti-tumor phenotype [129].

\section{Neutrophil effects on tumor and immune cells}

TANs can play dichotomous roles in the context of tumor progression, alternatively functioning in an anti-tumor and pro-tumor manner under the influence of complex TME conditions [134]. Below, we primarily discuss the ability of TANs to promote tumor progression (Figure 4).

\section{Reactive oxygen species}

Several lines of evidence suggest that neutrophils may be linked to carcinogenesis through ROS-dependent and -independent mechanisms. Owing to their robust phagocytic activity, neutrophils can produce high phagolysosomal levels of ROS that can aid in pathogen killing [135]. However, the release of these ROS by neutrophils can result in DNA damage and mutations that are linked to cancer initiation [136]. ROS can also result in epithelial damage and pro-oncogenic inflammation [128].

\section{Cytokines and chemokines}

Neutrophils release various cytokines and chemokines into the TME. Neutrophil-derived secreted factors primarily act in a pro-oncogenic manner. OSM is a pleiotropic cytokine in the IL-6 family that reportedly promotes tumor progression by enhancing angiogenesis and metastasis. When cocultured with breast cancer cells, neutrophils express and release high levels of OSM, which increases cancer cell detachment and invasive capacity [137]. Similarly, neutrophils cultivated in tumor-conditioned medium derived from HuCC-T1 or HepG2 cells secrete high levels of hepatocyte growth factor (HGF), which can increase the invasiveness of various cancer cell types, thus suggesting a possible role for TANs in tumor invasion [138]. Neutrophil recruitment into tumors appears to be dependent upon the binding of specific chemokines to CXCR1 and CXCR2 expressed on the surface of neutrophils. Inhibiting CXCR2 signaling can significantly reduce the numbers of tumor-infiltrating neutrophils both in vitro and in vivo in tumor model systems, and is associated with slower tumor growth [139]. In papillomas mouse models, CXCR2 deficiency can reduce tumor microvessel density by approximately $50 \%$ while reducing the size of tumors formed. Moreover, compared with adjacent normal tissues, papillomas upregulate several CXCR2 ligands, including CXCL1, CXCL2, and CXCL5 [140].

Neutrophil-derived secreted proteins can also disturb the functions of other immune cells. For example, OSM can promote M2 macrophage polarization in the TME in a manner dependent upon the mechanistic target of rapamycin kinase signaling complex 2 (mTORC2) [141].

\section{Enzymes}

Neutrophils contain four types of granules: primary (azurophil), secondary, and tertiary granules, as well as secretory vesicles. These granules contain a range of different proteases, with the best-studied of these being cathepsin $\mathrm{G}$ (CG), neutrophil elastase (NE), and MMP-9 [142]. These factors have been found to play a pro-metastatic role through mechanisms associated with the EMT and ECM remodeling [143]. CG is a serine protease that is presynthesized in promyelocytes in the bone marrow and then stored in neutrophil primary granules in an active protease form. In breast cancer MCF-7 cells, CG can bind to the tumor cell surface in a manner independent of its catalytic site, thereby inducing cell aggregation in a manner dependent upon its enzymatic activity [144]. The formation of these tumor cell aggregates can permit tumor cell dissemination through the circulatory system to distant sites and where new metastases can be established. The inhibition of CG was found to result in reduced osteolysis in breast cancer, highlighting CG as a potential therapeutic target in this oncogenic context [145]. NE is also a serine protease that is released upon neutrophil degranulation. NE can directly induce tumor cell proliferation in the context of both human and murine lung adenocarcinoma by gaining access to 
the endosomal compartment within tumor cells, wherein it degrades insulin receptor substrate-1 (IRS-1). IRS-1 degradation results in increased interactions between phosphatidylinositol 3-kinase (PI3K) and the potent mitogen platelet-derived growth factor receptor (PDGFR), thereby skewing the PI3K axis to favor tumor cell proliferation [146]. This pro-tumor function has also been observed in other tumor types including esophageal cancer, gastric cancer, and breast cancer [147-149]. In these cancer types, NE was found to mediate the release of transforming growth factoralpha (TGF- $\alpha$ ) from the cell surface. Higher levels of NE in breast cancer patients are correlated with lower survival rates, suggesting that $\mathrm{NE}$ is an independent prognostic biomarker in at least certain cancers [150]. NE and CG, as serine proteases, can also promote lung metastasis by degrading the anti-cancer protein thrombospondin 1 (Tsp1) in vitro and in vivo [151]. In addition to $\mathrm{NE}$ and CG, neutrophil-derived MMPs including MMP-8 and MMP-9 are associated with cancer progression [152]. Although MMP-9 is expressed in many cell types, studies suggest TANs are major contributors of highly angiogenic MMP-9 [153], and MMP-9 is stored in secondary granules in neutrophils wherein it is not associated with tissue inhibitor matrix metalloproteinase 1 (TIMP-1), rendering it better able to promote angiogenesis [147].

Enzymes secreted by neutrophils can influence the function of other immune cells. For example, tumorderived IL-1 $\beta$ activates $\gamma \delta \mathrm{T}$ cells to produce IL-17. IL17-producing $\gamma \delta \mathrm{T}$ cells can promote the upregulation of G-CSF in mammary tumors, leading to subsequent alterations in the neutrophil phenotypes discussed above. These phenotypically altered neutrophils can produce iNOS that suppresses the activity of anti-tumor $\mathrm{CD}^{+}$ $\mathrm{T}$ cells, thereby promoting breast cancer metastasis [154].

\section{Therapeutic targets in cancer}

Although there is substantial evidence that neutrophils play a deleterious role in tumor progression, therapeutically targeting this cell type in cancer remains very challenging. Neutrophils are critical mediators of host defenses against infection, and the depletion of these cells can thus result in dangerous levels of immunosuppression [152]. TANs themselves or specific neutrophil-promoting factors may represent potential immunotherapeutic targets (Table 2). One such promising therapeutic approach is the inhibition of CXCR2, which is the positive regulator of neutrophil mobilization. Very few tumor cells express CXCR2, suggesting that the effects of agents targeting this chemokine receptor are specifically attributable to immune cells. The combined inhibition of CXCR2 and PD-1 efficiently improves survival and suppresses metastasis, confirming the ability of CXCR2 to confer sensitivity to anti-PD-1 therapy [155]. In clinical trials, CXCR2 antagonists have mainly been used in patients with respiratory diseases such as chronic obstructive pulmonary disease and asthma $[156,157]$. Their therapeutic utility in cancer patients remains an area of active study.

Rather than targeting neutrophils directly, inhibiting specific neutrophil-derived enzymes known to promote tumor growth and invasiveness may also represent a viable anti-tumor treatment strategy. The function of $\mathrm{NE}$ in the context of tumor progression has been discussed in detail. However, recent NE-related clinical trials have focused on patients with bronchiectasis [158]. The application of these $\mathrm{NE}$ inhibitors to treat cancer patients largely sought to alleviate the side effects of other cancer treatments. For example, after receiving a NE inhibitor, patients with thoracic esophageal carcinoma showed an improved systemic inflammatory response [159]. Other therapies, such as inhibiting TGF- $\beta$ or targeting tumor necrosis factor-related apoptosisinducing ligand receptor (TRAIL-R) or SIRP $\alpha$ may also represent effective treatment strategies [160]. However, these signals can be detected by many other immune cells, and are not specific to TANs $[161,162]$. As such, we have elected not to discuss these pathways in detail in the present review. Overall, further research into the therapeutic utility of TANs is needed, as they are not as well studied as TAMs or DCs. Most importantly, additional preclinical and clinical studies are needed to better understand the therapeutic effects of targeting neutrophils in cancer patients.

In this review, we specially focus on their detrimental roles to the host by secreting cytokines and enzymes. Nevertheless, TAN anti-tumor function shouldn't be ignored. Since the incomplete research on exact roles, recruitment pathways, subpopulations and mechanisms of action of TANs, corresponding specific therapies remain to be developed.

\section{Concluding remarks}

With the recent and remarkable success in the development of novel cancer immunotherapies and the targeting of traditional $\mathrm{T}$ cells, there is an urgent need to elucidate the relationship between different immune components of the TME in order to improve the understanding of ICB and to establish reliable tumor biomarkers and effective combination treatment strategies. In this review, we have summarized key factors secreted by multiple myeloid cell types that can regulate or be affected by cell heterogeneity, 
cancer type, and individual differences. We further dissected the cross-talk between myeloid cells and other cell populations within the TME and provided clinical insights regarding the application of myeloid cells in the field of patient treatment.

Additional work is still necessary to identify specific cytokines with potential therapeutic utility. With respect to cellular interplay, a majority of studies to date have focused on the ability of tumor cells to reprogram other cell types, with the interactions between different immune cells and between immune and non-immune cells remaining less well studied. Moreover, most in vitro experiments consist of two kinds of cells exposed to specific culture conditions, thus failing to recapitulate the true complexity of the TME. Recent developments in the generation of three-dimensional (3D) tumor models offer a better opportunity to mimic the TME, and thus warrant broad application [163]. There is now substantial evidence to support the utility of myeloid-based targeted therapies that can impact solid tumor progression and provide clinical survival benefits. New checkpoints associated with different types of cells in the immune system have the potential to further advance the field of immunotherapy. Precisely eliminating the pro-tumor myeloid cells within the TME may represent another viable therapeutic strategy. Although techniques such as the CIBERSORT and XCell algorithms can estimate the abundance of tumor-infiltrating myeloid cells by using gene expression data from bulk tissues and flow cytometry in order to highlight the complexity of major myeloid lineages, these approaches are insufficiently detailed or scalable to permit effective phenotypic differentiation [164, 165]. With recent advances in the development of single-cell RNA sequencing technologies, more interesting myeloid cell subtypes have been identified, that may explain the variable efficacy of certain myeloid-targeted antibodies [16]. Considering the challenges associated with capturing and identifying immune checkpoints and immune cell populations in transcriptomic datasets, further technologies and approaches will be necessary to validate these observations.

Future research efforts should seek to clarify which myeloid cell subtypes are crucial to human disease, to elucidate their functional roles, and to establish whether they can be manipulated in precision medicine applications. Toxicity is an additional important consideration in the context of myeloid-based targeted therapy, and it remains very challenging to patient samples with immune-related adverse events (irAEs) in clinical trials. As little is known regarding the mechanistic basis for such irAEs, it may be difficult to link these myeloid-based targeted therapies with irAE incidence. Further studies also need assess the safety of myeloid-based targeted therapy. In addition, cancer is a systemic disease that affects the entire immune system. In addition to focusing on local immune responses within the TME, we must assess the systemic immune landscape. As such, it is necessary to further study the mechanisms underlying myeloid-based targeted therapies in order to evaluate the safety of these drugs, and to then conduct additional research and clinical trials.

Research funding: This work was supported by grants from the National Natural Science Foundation of China (No. 82073145 to Y.Y.), Shanghai Pujiang Program (No. 20PJ1412800 to Y.Y.), and Natural Science Foundation of Shanghai (No. 20ZR1472900 to Y.Y.).

Author contributions: Y.Y. conceived and supervised the project. M.S. and Y.Y. collected all related literatures, designed schematic diagram and interpreted results. M.S., Y.D., and Y.Y. wrote the manuscript. All authors have accepted responsibility for the entire content of this manuscript and approved its submission.

Competing interests: Authors state no conflict of interest. Ethical approval: The local Institutional Review Board deemed the study exempt from review.

\section{References}

1. Engblom C, Pfirschke C, Pittet MJ. The role of myeloid cells in cancer therapies. Nat Rev Cancer 2016;16:447-62.

2. Duan Q, Zhang H, Zheng J, Zhang L. Turning cold into hot: firing up the tumor microenvironment. Trends Cancer 2020;6:605-18.

3. Binnewies M, Roberts EW, Kersten K, Chan V, Fearon DF, Merad $M$, et al. Understanding the tumor immune microenvironment (TIME) for effective therapy. Nat Med 2018;24:541-50.

4. Sharma $P$, Allison JP. The future of immune checkpoint therapy. Science 2015;348:56-61.

5. Nakamura K, Smyth MJ. Myeloid immunosuppression and immune checkpoints in the tumor microenvironment. Cell Mol Immunol 2020;17:1-12.

6. Mantovani A, Marchesi F, Malesci A, Laghi L, Allavena P. Tumourassociated macrophages as treatment targets in oncology. Nat Rev Clin Oncol 2017;14:399-416.

7. Wculek SK, Cueto FJ, Mujal AM, Melero I, Krummel MF, Sancho D. Dendritic cells in cancer immunology and immunotherapy. Nat Rev Immunol 2020;20:7-24.

8. Wynn TA, Chawla A, Pollard JW. Macrophage biology in development, homeostasis and disease. Nature 2013;496:445-55.

9. Lavin Y, Mortha A, Rahman A, Merad M. Regulation of macrophage development and function in peripheral tissues. Nat Rev Immunol 2015;15:731-44.

10. Ginhoux F, Guilliams M. Tissue-resident macrophage ontogeny and homeostasis. Immunity 2016;44:439-49. 
11. Zhu Y, Herndon JM, Sojka DK, Kim KW, Knolhoff BL, Zuo C, et al. Tissue-resident macrophages in pancreatic ductal adenocarcinoma originate from embryonic hematopoiesis and promote tumor progression. Immunity 2017;47:323-38.e326.

12. Zhang L, Li Z, Skrzypczynska KM, Fang Q, Zhang W, O’Brien SA, et al. Single-cell analyses inform mechanisms of myeloidtargeted therapies in colon cancer. Cell 2020;181:442-59.e29.

13. La Manno G, Soldatov R, Zeisel A, Braun E, Hochgerner H, Petukhov V, et al. RNA velocity of single cells. Nature 2018;560: 494-8.

14. Zhou J, Tang Z, Gao S, Li C, Feng Y, Zhou X. Tumor-associated macrophages: recent insights and therapies. Front Oncol 2020; 10:188.

15. Mantovani A, Sozzani S, Locati M, Allavena P, Sica A. Macrophage polarization: tumor-associated macrophages as a paradigm for polarized M2 mononuclear phagocytes. Trends Immunol 2002;23:549-55.

16. Cheng S, Li Z, Gao R, Xing B, Gao Y, Yang Y, et al. A pan-cancer single-cell transcriptional atlas of tumor infiltrating myeloid cells. Cell 2021;184:792-809.

17. Qu Y, Wen J, Thomas G, Yang W, Prior W, He W, et al. Baseline frequency of inflammatory Cxcl9-expressing tumor-associated macrophages predicts response to avelumab treatment. Cell Rep 2020;32:107873.

18. Zhao P, Gao D, Wang Q, Song B, Shao Q, Sun J, et al. Response gene to complement 32 (RGC-32) expression on M2-polarized and tumor-associated macrophages is M-CSF-dependent and enhanced by tumor-derived IL-4. Cell Mol Immunol 2015;12: 692-9.

19. Ma R, Ji T, Chen D, Dong W, Zhang H, Yin X, et al. Tumor cellderived microparticles polarize $M 2$ tumor-associated macrophages for tumor progression. Oncoimmunology 2016;5: e1118599.

20. Huber R, Meier B, Otsuka A, Fenini G, Satoh T, Gehrke S, et al. Tumour hypoxia promotes melanoma growth and metastasis via high mobility group box-1 and M2-like macrophages. Sci Rep 2016;6:29914.

21. Tripathi C, Tewari BN, Kanchan RK, Baghel KS, Nautiyal N, Shrivastava R, et al. Macrophages are recruited to hypoxic tumor areas and acquire a pro-angiogenic M2-polarized phenotype via hypoxic cancer cell derived cytokines oncostatin $M$ and eotaxin. Oncotarget 2014;5:5350-68.

22. Ambade A, Satishchandran A, Saha B, Gyongyosi B, Lowe P, Kodys $\mathrm{K}$, et al. Hepatocellular carcinoma is accelerated by NASH involving $\mathrm{M} 2$ macrophage polarization mediated by hif1ainduced IL-10. Oncoimmunology 2016;5:e1221557.

23. DeNardo DG, Ruffell B. Macrophages as regulators of tumour immunity and immunotherapy. Nat Rev Immunol 2019;19: 369-82.

24. Henze AT, Mazzone M. The impact of hypoxia on tumorassociated macrophages. J Clin Invest 2016;126:3672-9.

25. Liu PS, Wang H, Li X, Chao T, Teav T, Christen S, et al. $\alpha$-ketoglutarate orchestrates macrophage activation through metabolic and epigenetic reprogramming. Nat Immunol 2017; 18:985-94.

26. Haskó G, Pacher P. Regulation of macrophage function by adenosine. Arterioscler Thromb Vasc Biol 2012;32:865-9.

27. Cekic C, Day YJ, Sag D, Linden J. Myeloid expression of adenosine a2A receptor suppresses T and NK cell responses in the solid tumor microenvironment. Cancer Res 2014;74:7250-9.
28. Colegio OR, Chu NQ, Szabo AL, Chu T, Rhebergen AM, Jairam V, et al. Functional polarization of tumour-associated macrophages by tumour-derived lactic acid. Nature 2014;513: 559-63.

29. Chen P, Zuo H, Xiong H, Kolar MJ, Chu Q, Saghatelian A, et al. Gpr132 sensing of lactate mediates tumor-macrophage interplay to promote breast cancer metastasis. Proc Natl Acad Sci USA 2017;114:580-5.

30. Lin Y, Xu J, Lan H. Tumor-associated macrophages in tumor metastasis: biological roles and clinical therapeutic applications. J Hematol Oncol 2019;12:76.

31. Yang L, Zhang Y. Tumor-associated macrophages: from basic research to clinical application. J Hematol Oncol 2017;10:58.

32. Savagner P. The epithelial-mesenchymal transition (EMT) phenomenon. Ann Oncol 2010;21(7 Suppl):vii89-92.

33. Lamouille S, Xu J, Derynck R. Molecular mechanisms of epithelial-mesenchymal transition. Nat Rev Mol Cell Biol 2014; 15:178-96.

34. Wei C, Yang C, Wang S, Shi D, Zhang C, Lin X, et al. Crosstalk between cancer cells and tumor associated macrophages is required for mesenchymal circulating tumor cell-mediated colorectal cancer metastasis. Mol Cancer 2019;18:64.

35. Fu XT, Dai Z, Song K, Zhang ZJ, Zhou ZJ, Zhou SL, et al. Macrophage-secreted IL-8 induces epithelial-mesenchymal transition in hepatocellular carcinoma cells by activating the JAK2/STAT3/snail pathway. Int J Oncol 2015;46:587-96.

36. Su S, Liu Q, Chen J, Chen J, Chen F, He C, et al. A positive feedback loop between mesenchymal-like cancer cells and macrophages is essential to breast cancer metastasis. Cancer Cell 2014;25: 605-20.

37. Chen $\mathrm{Y}$, Tan W, Wang C. Tumor-associated macrophage-derived cytokines enhance cancer stem-like characteristics through epithelial-mesenchymal transition. Onco Targets Ther 2018;11: 3817-26.

38. Poltavets V, Kochetkova M, Pitson SM, Samuel MS. The role of the extracellular matrix and its molecular and cellular regulators in cancer cell plasticity. Front Oncol 2018;8:431.

39. Chen J, Yao Y, Gong C, Yu F, Su S, Chen J, et al. CCL18 from tumorassociated macrophages promotes breast cancer metastasis via PITPNM3. Cancer Cell 2011;19:541-55.

40. Sharma SK, Chintala NK, Vadrevu SK, Patel J, Karbowniczek M, Markiewski MM. Pulmonary alveolar macrophages contribute to the premetastatic niche by suppressing antitumor T cell responses in the lungs. J Immunol 2015;194:5529-38.

41. Kimura Y, Inoue A, Hangai S, Saijo S, Negishi H, Nishio J, et al. The innate immune receptor dectin-2 mediates the phagocytosis of cancer cells by Kupffer cells for the suppression of liver metastasis. Proc Natl Acad Sci USA 2016;113:14097-102.

42. Lin EY, Li JF, Gnatovskiy L, Deng Y, Zhu L, Grzesik DA, et al. Macrophages regulate the angiogenic switch in a mouse model of breast cancer. Cancer Res 2006;66:11238-46.

43. Lin EY, Pollard JW. Tumor-associated macrophages press the angiogenic switch in breast cancer. Cancer Res 2007;67: 5064-6.

44. Huang S, Van Arsdall M, Tedjarati S, McCarty M, Wu W, Langley $R$, et al. Contributions of stromal metalloproteinase- 9 to angiogenesis and growth of human ovarian carcinoma in mice. J Natl Cancer Inst 2002;94:1134-42.

45. Riabov V, Gudima A, Wang N, Mickley A, Orekhov A, Kzhyshkowska J. Role of tumor associated macrophages in 
tumor angiogenesis and lymphangiogenesis. Front Physiol 2014;5:75.

46. Lewis CE, De Palma M, Naldini L. Tie2-expressing monocytes and tumor angiogenesis: regulation by hypoxia and angiopoietin-2. Cancer Res 2007;67:8429-32.

47. Huang H, Lai JY, Do J, Liu D, Li L, Del Rosario J, et al. Specifically targeting angiopoietin-2 inhibits angiogenesis, Tie2-expressing monocyte infiltration, and tumor growth. Clin Cancer Res 2011; 17:1001-11.

48. Matsubara T, Kanto T, Kuroda S, Yoshio S, Higashitani K, Kakita N, et al. TIE2-expressing monocytes as a diagnostic marker for hepatocellular carcinoma correlates with angiogenesis. Hepatology 2013;57:1416-25.

49. Raskov H, Orhan A, Christensen JP, Gögenur I. Cytotoxic CD8 ${ }^{+} \mathrm{T}$ cells in cancer and cancer immunotherapy. Br J Cancer 2021;124: 359-67.

50. Tanaka A, Sakaguchi S. Regulatory T cells in cancer immunotherapy. Cell Res 2017;27:109-18.

51. Ruffell B, Chang-Strachan D, Chan V, Rosenbusch A, Ho CMT, Pryer N, et al. Macrophage IL-10 blocks $\mathrm{CD} 8^{+} \mathrm{T}$ cell-dependent responses to chemotherapy by suppressing IL-12 expression in intratumoral dendritic cells. Cancer Cell 2014;26:623-37.

52. Sun X, He X, Zhang Y, Hosaka K, Andersson P, Wu J, et al. Inflammatory cell-derived CXCL3 promotes pancreatic cancer metastasis through a novel myofibroblast-hijacked cancer escape mechanism. Gut 2021. https://doi.org/10.1136/gutjnl2020-322744.

53. Hume DA, MacDonald KPA. Therapeutic applications of macrophage colony-stimulating factor-1 (CSF-1) and antagonists of CSF-1 receptor (CSF-1R) signaling. Blood 2012;119:1810-20.

54. Ries CH, Cannarile MA, Hoves S, Benz J, Wartha K, Runza V, et al. Targeting tumor-associated macrophages with anti-CSF-1R antibody reveals a strategy for cancer therapy. Cancer Cell 2014; 25:846-59.

55. Cannarile MA, Weisser M, Jacob W, Jegg AM, Ries CH, Rüttinger D. Colony-stimulating factor 1 receptor (CSF1R) inhibitors in cancer therapy. J Immunother Cancer 2017;5:53.

56. Qian BZ, Li J, Zhang H, Kitamura T, Zhang J, Campion LR, et al. CCL2 recruits inflammatory monocytes to facilitate breasttumour metastasis. Nature 2011;475:222-5.

57. Nywening TM, Wang-Gillam A, Sanford DE, Belt BA, Panni RZ, Cusworth BM, et al. Targeting tumour-associated macrophages with CCR2 inhibition in combination with FOLFIRINOX in patients with borderline resectable and locally advanced pancreatic cancer: a single-centre, open-label, dose-finding, nonrandomised, phase $1 \mathrm{~b}$ trial. Lancet Oncol 2016;17:651-62.

58. Linehan D, Noel MS, Hezel AF, Wang-Gillam A, Eskens F, Sleijfer $S$, et al. Overall survival in a trial of orally administered CCR2 inhibitor CCX872 in locally advanced/metastatic pancreatic cancer: correlation with blood monocyte counts. J Clin Oncol 2018;36:92.

59. Bonapace L, Coissieux MM, Wyckoff J, Mertz KD, Varga Z, Junt T, et al. Cessation of CCL2 inhibition accelerates breast cancer metastasis by promoting angiogenesis. Nature 2014;515:130-3.

60. Franciszkiewicz K, Boissonnas $A$, Boutet $M$, Combadière $C$, Mami-Chouaib F. Role of chemokines and chemokine receptors in shaping the effector phase of the antitumor immune response. Cancer Res 2012;72:6325-32.

61. Beatty GL, Chiorean EG, Fishman MP, Saboury B, Teitelbaum UR, Sun W, et al. CD40 agonists alter tumor stroma and show efficacy against pancreatic carcinoma in mice and humans. Science 2011;331:1612-6.

62. Winograd R, Byrne KT, Evans RA, Odorizzi PM, Meyer ARL, Bajor DL, et al. Induction of T-cell immunity overcomes complete resistance to PD-1 and CTLA- 4 blockade and improves survival in pancreatic carcinoma. Cancer Immunol Res 2015;3:399-411.

63. Chan GCF, Chan WK, Sze DMY. The effects of beta-glucan on human immune and cancer cells. J Hematol Oncol 2009;2:25.

64. Barkal AA, Brewer RE, Markovic M, Kowarsky M, Barkal SA, Zaro BW, et al. CD24 signalling through macrophage siglec-10 is a target for cancer immunotherapy. Nature 2019;572:392-6.

65. Song Q, Hawkins GA, Wudel L, Chou PC, Forbes E, Pullikuth AK, et al. Dissecting intratumoral myeloid cell plasticity by single cell RNA-seq. Cancer Med 2019;8:3072-85.

66. Lavin Y, Kobayashi S, Leader A, Amir ED, Elefant N, Bigenwald C, et al. Innate immune landscape in early lung adenocarcinoma by paired single-cell analyses. Cell 2017;169:750-65.e17.

67. Molgora M, Esaulova E, Vermi W, Hou J, Chen Y, Luo J, et al. TREM2 modulation remodels the tumor myeloid landscape enhancing anti-PD-1 immunotherapy. Cell 2020;182: 886-900.e17.

68. Noubade R, Majri-Morrison S, Tarbell KV. Beyond CDC1: emerging roles of DC crosstalk in cancer immunity. Front Immunol 2019;10:1014.

69. Anderson DA, Dutertre CA, Ginhoux F, Murphy KM. Genetic models of human and mouse dendritic cell development and function. Nat Rev Immunol 2021;21:101-15.

70. Veglia F, Gabrilovich DI. Dendritic cells in cancer: the role revisited. Curr Opin Immunol 2017;45:43-51.

71. Soares H, Waechter HN, Glaichenhaus N, Mougneau E, Yagita H, Mizenina 0 , et al. A subset of dendritic cells induces $C D 4^{+} T$ cells to produce IFN- $\gamma$ by an IL-12-independent but CD70-dependent mechanism in vivo. J Exp Med 2007;204:1095-106.

72. Broz ML, Binnewies M, Boldajipour B, Nelson AE, Pollack JL, Erle DJ, et al. Dissecting the tumor myeloid compartment reveals rare activating antigen-presenting cells critical for T cell immunity. Cancer Cell 2014;26:638-52.

73. Romagnani S. Th1/Th2 cells. Inflamm Bowel Dis 1999;5:285-94.

74. Binnewies M, Mujal AM, Pollack JL, Combes AJ, Hardison EA, Barry KC, et al. Unleashing type-2 dendritic cells to drive protective antitumor $\mathrm{CD}^{+}{ }^{+}$cell immunity. Cell 2019;177: 556-71.

75. Domínguez PM, Ardavín C. Differentiation and function of mouse monocyte-derived dendritic cells in steady state and inflammation. Immunol Rev 2010;234:90-104.

76. Brown CC, Gudjonson H, Pritykin Y, Deep D, Lavallée VP, Mendoza A, et al. Transcriptional basis of mouse and human dendritic cell heterogeneity. Cell 2019;179:846-63.

77. Hanks BA, Holtzhausen A, Evans KS, Jamieson R, Gimpel P, Campbell OM, et al. Type III TGF- $\beta$ receptor downregulation generates an immunotolerant tumor microenvironment. J Clin Invest 2013;123:3925-40.

78. Zong J, Keskinov AA, Shurin GV, Shurin MR. Tumor-derived factors modulating dendritic cell function. Cancer Immunol Immunother 2016;65:821-33.

79. Ferrara N, Gerber HP, LeCouter J. The biology of VEGF and its receptors. Nat Med 2003;9:669-76.

80. Gabrilovich DI, Chen HL, Girgis KR, Cunningham HT, Meny GM, Nadaf S, et al. Production of vascular endothelial growth factor 
by human tumors inhibits the functional maturation of dendritic cells. Nat Med 1996;2:1096-103.

81. Kim R, Emi M, Tanabe K, Arihiro K. Tumor-driven evolution of immunosuppressive networks during malignant progression. Cancer Res 2006;66:5527-36.

82. Williams LM, Ricchetti G, Sarma U, Smallie T, Foxwell BMJ. Interleukin-10 suppression of myeloid cell activation - a continuing puzzle. Immunology 2004;113:281-92.

83. Kim KD, Lim HY, Lee HG, Yoon DY, Choe YK, Choi I, et al. Apolipoprotein A-I induces IL-10 and PGE2 production in human monocytes and inhibits dendritic cell differentiation and maturation. Biochem Biophys Res Commun 2005;338:1126-36.

84. Nefedova Y, Huang M, Kusmartsev S, Bhattacharya R, Cheng P, Salup R, et al. Hyperactivation of STAT3 is involved in abnormal differentiation of dendritic cells in cancer. J Immunol 2004;172: 464-74.

85. Aalamian M, Tourkova IL, Chatta GS, Lilja H, Huland E, Huland H, et al. Inhibition of dendropoiesis by tumor derived and purified prostate specific antigen. J Urol 2003;170:2026-30.

86. Monti P, Leone BE, Zerbi A, Balzano G, Cainarca S, Sordi V, et al. Tumor-derived MUC1 mucins interact with differentiating monocytes and induce IL-10 high IL-12 low regulatory dendritic cell. J Immunol 2004;172:7341-9.

87. Carlos CA, Dong HF, Howard OMZ, Oppenheim JJ, Hanisch F-G, Finn 0 J. Human tumor antigen MUC1 is chemotactic for immature dendritic cells and elicits maturation but does not promote Th1 type immunity. J Immunol 2005;175:1628-35.

88. Giovanelli P, Sandoval TA, Cubillos-Ruiz JR. Dendritic cell metabolism and function in tumors. Trends Immunol 2019;40: 699-718.

89. Gottfried E, Kunz-Schughart LA, Ebner S, Mueller-Klieser W, Hoves S, Andreesen R, et al. Tumor-derived lactic acid modulates dendritic cell activation and antigen expression. Blood 2006;107:2013-21.

90. Munn DH, Mellor AL. IDO in the tumor microenvironment: inflammation, counter-regulation, and tolerance. Trends Immunol 2016;37:193-207.

91. Beloribi-Djefaflia S, Vasseur S, Guillaumond F. Lipid metabolic reprogramming in cancer cells. Oncogenesis 2016;5:e189.

92. Herber DL, Cao W, Nefedova Y, Novitskiy SV, Nagaraj S, Tyurin VA, et al. Lipid accumulation and dendritic cell dysfunction in cancer. Nat Med. 2010;16:880-6.

93. Zhao F, Xiao C, Evans KS, Theivanthiran T, DeVito N, Holtzhausen $A$, et al. Paracrine Wnt5a- $\beta$-catenin signaling triggers a metabolic program that drives dendritic cell tolerization. Immunity 2018;48:147-60.e7.

94. Tong D, Liu Q, Wang L, Xie Q, Pang J, Huang Y, et al. The roles of the COX2/PGE2/EP axis in therapeutic resistance. Cancer Metastasis Rev 2018;37:355-68.

95. Greenhough A, Smartt HJM, Moore AE, Roberts HR, Williams AC, Paraskeva $C$, et al. The COX-2/PGE2 pathway: key roles in the hallmarks of cancer and adaptation to the tumour microenvironment. Carcinogenesis 2009;30:377-86.

96. Kalinski P. Regulation of immune responses by prostaglandin E 2 . J Immunol 2012;188:21-8.

97. Zelenay S, Van Der Veen AG, Böttcher JP, Snelgrove KJ, Rogers N, Acton SE, et al. Cyclooxygenase-dependent tumor growth through evasion of immunity. Cell 2015;162:1257-70.

98. Rowshanravan B, Halliday N, Sansom DM. CTLA-4: a moving target in immunotherapy. Blood 2018;131:58-67.
99. Peng Q, Qiu X, Zhang Z, Zhang S, Zhang Y, Liang Y, et al. PD-L1 on dendritic cells attenuates $T$ cell activation and regulates response to immune checkpoint blockade. Nat Commun 2020; 11:4835.

100. Cella M, Scheidegger D, Palmer-Lehmann K, Lane P, Lanzavecchia A, Alber G. Ligation of CD40 on dendritic cells triggers production of high levels of interleukin-12 and enhances T cell stimulatory capacity: T-T help via APC activation. J Exp Med 1996;184:747-52.

101. Smits HH, de Jong EC, Schuitemaker JHN, Geijtenbeek TBH, van Kooyk Y, Kapsenberg ML, et al. Intercellular adhesion molecule-1/LFA-1 ligation favors human Th1 development. J Immunol 2002;168:1710-6.

102. Dannull J, Nair S, Su Z, Boczkowski D, DeBeck C, Yang B, et al. Enhancing the immunostimulatory function of dendritic cells by transfection with mRNA encoding OX40 ligand. Blood 2005;105: 3206-13.

103. Bullock TNJ, Yagita H. Induction of CD70 on dendritic cells through CD40 or TLR stimulation contributes to the development of $\mathrm{CD}^{+} \mathrm{T}$ cell responses in the absence of $\mathrm{CD} 4^{+} \mathrm{T}$ cells. J Immunol 2005;174:710-7.

104. De Jong EC, Smits HH, Kapsenberg ML. Dendritic cell-mediated T cell polarization. Springer Semin Immunopathol 2005;26: 289-307.

105. Curtsinger JM, Mescher MF. Inflammatory cytokines as a third signal for T cell activation. Curr Opin Immunol 2010;22: 333-40.

106. Snijders A, Kalinski P, Hilkens CMU, Kapsenberg ML. High-level IL-12 production by human dendritic cells requires two signals. Int Immunol 1998;10:1593-8.

107. McRae BL, Semnani RT, Hayes MP, van Seventer GA. Type I IFNs inhibit human dendritic cell IL-12 production and Th1 cell development. J Immunol 1998;160:4298-304.

108. Landskron G, De La Fuente M, Thuwajit $P$, Thuwajit C, Hermoso MA. Chronic inflammation and cytokines in the tumor microenvironment. J Immunol Res 2014;2-14:149185.

109. Manicassamy S, Reizis B, Ravindran R, Nakaya H, SalazarGonzalez RM, Wang YC, et al. Activation of $\beta$-catenin in dendritic cells regulates immunity vs. tolerance in the intestine. Science 2010;329:849-53.

110. Liu G, Bi Y, Xue L, Zhang Y, Yang H, Chen X, et al. Dendritic cell SIRT1-HIF1 $\alpha$ axis programs the differentiation of $C D 4^{+}$T cells through IL-12 and TGF- $\beta 1$. Proc Natl Acad Sci USA 2015;112: E957-65.

111. Spranger S, Dai D, Horton B, Gajewski TF. Tumor-Residing Batf3 dendritic cells are required for effector $T$ cell trafficking and adoptive T cell therapy. Cancer Cell 2017;31:711-23.

112. Pfirschke C, Siwicki M, Liao H-W, Pittet MJ. Tumor microenvironment: no effector T cells without dendritic cells. Cancer Cell 2017;31:614-5.

113. Sabado RL, Balan S, Bhardwaj N. Dendritic cell-based immunotherapy. Cell Res 2017;27:74-95.

114. Mulder WJM, Ochando J, Joosten LAB, Fayad ZA, Netea MG. Therapeutic targeting of trained immunity. Nat Rev Drug Discov 2019;18:553-66.

115. Tong AW, Stone MJ. Prospects for CD40-directed experimental therapy of human cancer. Cancer Gene Ther 2003;10:1-13.

116. Vonderheide RH. The immune revolution: a case for priming, not checkpoint. Cancer Cell 2018;33:563-9. 
117. Beatty GL, Torigian DA, Gabriela Chiorean E, Saboury B, Brothers A, Alavi A, et al. A phase I study of an agonist CD40 monoclonal antibody (CP-870,893) in combination with gemcitabine in patients with advanced pancreatic ductal adenocarcinoma. Clin Cancer Res 2013;19:6286-95.

118. van Kooyk Y. C-type lectins on dendritic cells: key modulators for the induction of immune responses. Biochem Soc Trans 2008; 36:1478-81.

119. Tullett KM, Leal Rojas IM, Minoda Y, Tan PS, Zhang J-G, Smith C, et al. Targeting CLEC9A delivers antigen to human CD141 ${ }^{+}$DC for $\mathrm{CD}^{+}$and $\mathrm{CD} 8^{+} \mathrm{T}$ cell recognition. JCI Insight 2016;1:e87102.

120. Dhodapkar MV, Sznol M, Zhao B, Wang D, Carvajal RD, Keohan $M L$, et al. Induction of antigen-specific immunity with a vaccine targeting NY-ESO-1 to the dendritic cell receptor DEC-205. Sci Transl Med 2014;6:232ra51.

121. Meadows KL, Hurwitz HI. Anti-VEGF therapies in the clinic. Cold Spring Harb Perspect Med 2012;2:a006577.

122. Gabrilovich DI, Ishida T, Nadaf S, Ohm JE, Carbone DP. Antibodies to vascular endothelial growth factor enhance the efficacy of cancer immunotherapy by improving endogenous dendritic cell function. Clin Cancer Res 1999;5:2963-70.

123. Osada T, Chong G, Tansik R, Hong T, Spector N, Kumar R, et al. The effect of anti-VEGF therapy on immature myeloid cell and dendritic cells in cancer patients. Cancer Immunol Immunother 2008;57:1115-24.

124. Nefedova Y, Cheng P, Gilkes D, Blaskovich M, Beg AA, Sebti SM, et al. Activation of dendritic cells via inhibition of Jak2/STAT3 signaling. J Immunol 2005;175:4338-46.

125. Johnson DE, O'Keefe RA, Grandis JR. Targeting the IL-6/JAK/STAT3 signalling axis in cancer. Nat Rev Clin Oncol 2018;15:234-48.

126. Jing H, Vassiliou E, Ganea D. Prostaglandin E2 inhibits production of the inflammatory chemokines CCL3 and CCL4 in dendritic cells. J Leukoc Biol 2003;74:868-79.

127. Moon YW, Hajjar J, Hwu P, Naing A. Targeting the indoleamine 2,3-dioxygenase pathway in cancer. J Immunother Cancer 2015;3: 1-10.

128. Coffelt SB, Wellenstein MD, de Visser KE. Neutrophils in cancer: neutral no more. Nat Rev Cancer 2016;16:431-46.

129. Fridlender ZG, Sun J, Kim S, Kapoor V, Cheng G, Ling L, et al. Polarization of tumor-associated neutrophil phenotype by TGF- $\beta$ : “N1" vs. “N2" TAN. Cancer Cell 2009;16:183-94.

130. Sagiv JY, Michaeli J, Assi S, Mishalian I, Kisos H, Levy L, et al. Phenotypic diversity and plasticity in circulating neutrophil subpopulations in cancer. Cell Rep 2015;10:562-73.

131. Luoma AM, Suo S, Williams HL, Sharova T, Sullivan K, Manos M, et al. Molecular pathways of colon inflammation induced by cancer immunotherapy. Cell 2020;182:655-71.e22.

132. Zilionis R, Engblom C, Pfirschke C, Savova V, Zemmour D, Saatcioglu HD, et al. Single-cell transcriptomics of human and mouse lung cancers reveals conserved myeloid populations across individuals and species. Immunity 2019;50:1317-34.e10.

133. Casbon AJ, Reynau D, Park C, Khu E, Gan DD, Schepers K, et al. Invasive breast cancer reprograms early myeloid differentiation in the bone marrow to generate immunosuppressive neutrophils. Proc Natl Acad Sci USA 2015;112:e566-75.

134. Uribe-Querol E, Rosales C. Neutrophils in cancer: two sides of the same coin. J Immunol Res 2015;2015:983698.

135. Winterbourn CC, Kettle AJ, Hampton MB. Reactive oxygen species and neutrophil function. Annu Rev Biochem 2016;85:765-92.
136. Cooke MS, Evans MD, Dizdaroglu M, Lunec J. Oxidative DNA damage: mechanisms, mutation, and disease. Faseb J 2003;17: 1195-214.

137. Queen MM, Ryan RE, Holzer RG, Keller-Peck CR, Jorcyk CL. Breast cancer cells stimulate neutrophils to produce oncostatin $M$ : potential implications for tumor progression. Cancer Res 2005; 65:8896-904.

138. Imai Y, Kubota Y, Yamamoto S, Tsuji K, Shimatani M, Shibatani $\mathrm{N}$, et al. Neutrophils enhance invasion activity of human cholangiocellular carcinoma and hepatocellular carcinoma cells: an in vitro study. J Gastroenterol Hepatol 2005;20:287-93.

139. Tazzyman S, Barry ST, Ashton S, Wood P, Blakey D, Lewis CE, et al. Inhibition of neutrophil infiltration into A549 lung tumors in vitro and in vivo using a CXCR2-specific antagonist is associated with reduced tumor growth. Int J Cancer 2011;129:847-58.

140. Jamieson T, Clarke M, Steele CW, Samuel MS, Neumann J, Jung A, et al. Inhibition of CXCR2 profoundly suppresses inflammationdriven and spontaneous tumorigenesis. J Clin Invest 2012;122: 3127-44.

141. Shrivastava R, Asif M, Singh V, Dubey P, Ahmad Malik S, Lone MUD, et al. M2 polarization of macrophages by Oncostatin $M$ in hypoxic tumor microenvironment is mediated by mTORC2 and promotes tumor growth and metastasis. Cytokine 2019;118: 130-43.

142. Borregaard N, Cowland JB. Granules of the human neutrophilic polymorphonuclear leukocyte. Blood 1997;89:3503-21.

143. Felix K, Gaida MM. Neutrophil-derived proteases in the microenvironment of pancreatic cancer-active players in tumor progression. Int J Biol Sci 2016;12:302-13.

144. Morimoto-Kamata R, Mizoguchi SI, Ichisugi T, Yui S. Cathepsin G induces cell aggregation of human breast cancer MCF-7 cells via a 2-step mechanism: catalytic site-independent binding to the cell surface and enzymatic activity-dependent induction of the cell aggregation. Mediat Inflamm 2012;2012:456462.

145. Wilson TJ, Nannuru KC, Futakuchi M, Sadanandam A, Singh RK. Cathepsin $\mathrm{G}$ enhances mammary tumor-induced osteolysis by generating soluble receptor activator of nuclear factor- $\mathrm{kB}$ ligand. Cancer Res 2008;68:5803-11.

146. Houghton AMG, Rzymkiewicz DM, Ji H, Gregory AD, Egea EE, Metz HE, et al. Neutrophil elastase-mediated degradation of IRS-1 accelerates lung tumor growth. Nat Med 2010;16: 219-23.

147. Wada Y, Yoshida K, Tsutani Y, Shigematsu H, Oeda M, Sanada Y, et al. Neutrophil elastase induces cell proliferation and migration by the release of TGF- $\alpha$, PDGF and VEGF in esophageal cell lines. Oncol Rep 2007;17:161-7.

148. Wada Y, Yoshida K, Hihara J, Konishi K, Tanabe K, Ukon K, et al. Sivelestat, a specific neutrophil elastase inhibitor, suppresses the growth of gastric carcinoma cells by preventing the release of transforming growth factor- $\alpha$. Cancer Sci 2006;97:1037-43.

149. Nawa M, Osada S, Morimitsu K, Nonaka K, Futamura M, Kawaguchi Y, et al. Growth effect of neutrophil elastase on breast cancer: favorable action of sivelestat and application to anti-HER2 therapy. Anticancer Res 2012;32:13-9.

150. Akizuki M, Fukutomi T, Takasugi M, Takahashi S, Sato T, Harao $M$, et al. Prognostic significance of immunoreactive neutrophil elastase in human breast cancer: long-term follow-up results in 313 patients. Neoplasia 2007;9:260-4. 
151. Rayes TE, Catena R, Lee S, Stawowczyk M, Joshi N, Fischbach C, et al. Lung inflammation promotes metastasis through neutrophil protease-mediated degradation of Tsp-1. Proc Natl Acad Sci USA 2015;112:16000-5.

152. Gregory AD, Houghton AMG. Tumor-associated neutrophils: new targets for cancer therapy. Cancer Res 2011;71:2411-6.

153. Ardi VC, Kupriyanova TA, Deryugina El, Quigley JP. Human neutrophils uniquely release TIMP-free MMP-9 to provide a potent catalytic stimulator of angiogenesis. Proc Natl Acad Sci USA 2007;104:20262-7.

154. Coffelt SB, Kersten K, Doornebal CW, Weiden J, Vrijland K, Hau CS, et al. IL-17-producing $\gamma \delta T$ cells and neutrophils conspire to promote breast cancer metastasis. Nature 2015; 522:345-8.

155. Steele CW, Karim SA, Leach JDG, Bailey P, Upstill-Goddard R, Rishi L, et al. CXCR2 inhibition profoundly suppresses metastases and augments immunotherapy in pancreatic ductal adenocarcinoma. Cancer Cell 2016;29:832-45.

156. O’Byrne PM, Metev H, Puu M, Richter K, Keen C, Uddin M, et al. Efficacy and safety of a CXCR2 antagonist, AZD5069, in patients with uncontrolled persistent asthma: a randomised, doubleblind, placebo-controlled trial. Lancet Respir Med 2016;4: 797-806.

157. Lazaar AL, Miller BE, Donald AC, Keeley T, Ambery C, Russell J, et al. CXCR2 antagonist for patients with chronic obstructive pulmonary disease with chronic mucus hypersecretion: a phase 2b trial. Respir Res 2020;21:149.

158. Stockley R, De Soyza A, Gunawardena K, Perrett J, ForsmanSemb K, Entwistle N, et al. Phase II study of a neutrophil elastase inhibitor (AZD9668) in patients with bronchiectasis. Respir Med 2013;107:524-33.

159. Suda K, Kitagawa Y, Ozawa S, Miyasho T, Okamoto M, Saikawa $\mathrm{Y}$, et al. Neutrophil elastase inhibitor improves postoperative clinical courses after thoracic esophagectomy. Dis Esophagus 2007;20:478-86.

160. Shaul ME, Fridlender ZG. Tumour-associated neutrophils in patients with cancer. Nat Rev Clin Oncol 2019;16:601-20.

161. Von Karstedt S, Montinaro A, Walczak H. Exploring the TRAILs less travelled: TRAIL in cancer biology and therapy. Nat Rev Cancer 2017;17:352-66.

162. Veillette A, Chen J. SIRP $\alpha-C D 47$ immune checkpoint blockade in anticancer therapy. Trends Immunol 2018;39:173-84.

163. Asghar W, El Assal R, Shafiee H, Pitteri S, Paulmurugan R, Demirci U. Engineering cancer microenvironments for in vitro 3-D tumor models. Mater Today 2015;18:539-53.

164. Newman AM, Liu CL, Green MR, Gentles AJ, Feng W, Xu Y, et al. Robust enumeration of cell subsets from tissue expression profiles. Nat Methods 2015;12:453-7.

165. Aran D, Hu Z, Butte AJ. xCell: digitally portraying the tissue cellular heterogeneity landscape. Genome Biol 2017;18:220. 